Martin Skamletz

\title{
»Man hat diese Erweiterung des Tonumfanges seit ein paar Jahren an den Tasteninstrumenten sehr weit getrieben.« Der Umgang mit Grenzen beim späten Mozart und beim frühen Beethoven
}

Zwischen August und Oktober I803 trifft bei Ludwig van Beethoven ein Flügel von Sébastien Érard aus Paris ein, der gegenüber den in Wien zu dieser Zeit gebräuchlichen fünfoktavigen Instrumenten (mit Tasten von $\mathrm{F}_{1}$ bis $\mathrm{f}^{\prime \prime}$ ) einen nach oben bis zum c'"' erweiterten Tonumfang aufweist. ${ }^{\mathrm{I}}$ Joseph Haydn besitzt zwar schon seit I80I ein ähnliches Klavier von Érard, aber offenbar erst dasjenige von Beethoven, der sich gerade auf der Suche nach einem neuen Instrument befindet und in dieser Sache auch mit Wiener Klavierbauern in Verhandlung steht, stößt in Wien auf größeres Interesse. Überhaupt mögen derartige Impulse von außen in Gestalt von Instrumenten ungewohnter Bauart den Wiener Klavierbau nach I80o zusätzlich beeinflusst haben. Dessen Entwicklung hinsichtlich des Tonumfangs wird durch Gert Hecher folgendermaßen zusammengefasst: »Das I9. Jahrhundert beginnt noch mit dem Fünfoktaven-Umfang, der gelegentlich, bei Walter, bis $\mathrm{g}^{3}$, dann von Schantz bis a ${ }^{3}$ und um $\mathrm{I}_{805}$ von den meisten Herstellern bis $\mathrm{c}^{4}$ erweitert wird. $\aleph^{2}$

Der auf einem Tasteninstrument zur Verfügung stehende Tonumfang ist natürlich nur einer von vielen technischen Aspekten, die sich in dieser Zeit weiterentwickeln; dass er sich aber als besonders einflussreich auf die sich dadurch ebenfalls verändernden kompositorischen Möglichkeiten erweist, wird schon von den Zeitgenossen so wahrgenommen, wie I806 einem Beitrag in der neugegründeten Wiener Theater-Zeitung zu entnehmen ist:

»Nicht blos der Umfang der menschlichen Stimmen, der tiefsten und höchsten zusammen, genügten den Tonfreunden der neuren Zeiten [...]; man wünschte auch noch tiefere und höhere Töne, als die menschliche Stimme angiebt, zu hören, und so entstanden in der Tiefe die Kontratöne und in der Höhe die Supratöne über das dreygestrichene c.

Das Instrument befindet sich heute im Oberösterreichischen Landesmuseum in Linz, siehe (mit Abbildung) www.landesmuseum.at/de/video-news/videos-detail/hammerfluegel.html (alle Internetadressen in diesem Beitrag zuletzt abgerufen am 3. April 2019), außerdem Alfons Huber: Beethovens Erard-Flügel. Überlegungen zu seiner Restaurierung, in: Restauro 3 (I990), S. I8I-I88.

2 Gert Hecher: Designentwicklung und bautechnische Datierungsmöglichkeiten, in: Das Wiener Klavier bis I850. Bericht des Symposiums »Das Wiener Klavier bis I850«, hg. von Beatrix Darmstädter, Alfons Huber und Rudolf Hopfner, Tutzing 2007, S. I79-I94, hier S. I92; vgl. auch die tabellarischen Aufstellungen bei Michael Latcham: The Stringing, Scaling and Pitch of Hammerflügel Built in the Southern German and Viennese Traditions Ij80-I820, München/Salzburg 2000, Bd. 2, Tabellen I-7, S. 2-8. 
Man hat diese Erweiterung des Tonumfanges seit ein paar Jahren an den Tasteninstrumenten sehr weit getrieben, und diese dadurch ungemein vervollkommt. - Man bauet jetzt Tastenwerke von sechs vollen Oktaven, bis viergestrichen c, ja sogar bis f und darüber. Sie werden sehr gesucht theils weil man das Reichere und Seltnere liebt, theils weil sich ungemein viel auf solchen Instrumenten machen und ein ganz eigener mächtiger Effekt durch geübte und kluge Tonkünstlerhand auf ihnen hervorbringen läßt. Die neuen Komponisten setzen nicht selten ihre besten Sachen für diesen Tonumfang, und wahren Musikfreunden rath ich, wenn sie sich ein neues Pianoforte, sey es in Flügel oder Tafelform, anschaffen, eins von der erweiterten Tonleiterlänge zu wählen.«

Das erste Klavierwerk, das Beethoven nach Erhalt des Érard-Flügels im Verlauf des Jahres I804 »für diesen Tonumfang setzt«(ihn dabei jedoch nur bis zum a"' und nicht bis zum c'"' ausnutzt), die GrafWaldstein gewidmete Klaviersonate C-Dur op.53, gehört möglicherweise tatsächlich zu seinen bis dahin »besten Sachen«; außerdem ist die Fertigstellung des Klavierparts seines Konzerts c-Moll op.37, das er schon seit Ende der I79oer-Jahre in Arbeit hat, erst mit Blick auf das reale Vorhandensein eines solchen Instruments möglich: Die offizielle Uraufführung dieses Werks durch Beethovens Schüler Ferdinand Ries erfolgt im Sommer I804, nachdem Beethoven selbst im Frühjahr I803 noch eine im Solopart wohl über weite Strecken improvisierte Fassung öffentlich gespielt hat - auf was für einem Instrument, ist nicht überliefert. Die (dabei gar nicht besonders zahlreichen) bis ins c'"' hoch führenden Passagen im Klavierpart werden in der Originalausgabe der Stimmen im Verlag des Wiener Kunst- und Industrie-Comptoirs im Herbst I804 als Ossia-Versionen auf einem zusätzlichen System gedruckt. ${ }^{4}$

Welche Bedeutung der Érard-Flügel bei alldem für Beethoven wirklich hat, ob er ihn schätzt und ob er mit seiner ungewohnten Mechanik zurechtkommt (bis heute durchaus umstrittene Fragen, auf die Tilman Skowroneck die jüngsten und sehr überzeugenden Antworten gegeben hat), 5 spielt für die folgenden Betrachtungen keine entscheidende Rolle: Es geht hier nicht um konkrete Instrumente mit bestimmten baulichen und

3 B.: Uiber Musik und musikalische Instrumente unserer Zeit, in: Wiener Theater-Zeitung I (I806), Nr. II (23. September 1806), S. I67-169, hier S. I68. Vielen Dank an Klaus Pietschmann für den Hinweis auf diesen Text.

4 Vgl. Ludwig van Beethoven. Thematisch-bibliographisches Werkverzeichnis, hg. von Kurt Dorfmüller, Norbert Gertsch und Julia Ronge, München 20I4, Bd. I, S. 288-292 (op.53) und S. 2I4-22I (op.37); Grand Concerto pour le Pianoforte [...] par Louis van Beethoven, op.37. À Vienne au Bureau d'Arts et d'Industrie [I804] (PN 289); vgl. auch Leon Plantinga: Beethoven's Concertos. Historp, Stple, Performance, New York/ London I999, S. II3-I35 (On the origins of piano concerto no.3) und S.307-309 (Appendix II: On redating piano concerto no.3: A summary).

5 Tilman Skowroneck: Beethoven's Erard Piano. Its Influence on his Compositions and on Viennese Fortepiano Building, in: Early Music 30/4 (2002), S. 522-538, akt. und erw. in ders.: Beethoven the Pianist, Cambridge 20IO, S. 85-II5 (The I803 Érard grand piano). 
klanglichen Eigenschaften, ${ }^{6}$ sondern gewissermaßen nur um die Idee eines Instruments, das die bisherige Obergrenze des Tonumfangs erweitert und damit zusätzliche Töne zur Verfügung stellt, die dem Komponieren neue Möglichkeiten in der Gestaltung von melodischen, harmonischen und tonartlich-formalen Zusammenhängen bieten - und die der Komponist, den althergebrachten Respekt vor der Obergrenze des Tonumfangs f'" ablegend, nun auch in gedruckten, also für eine breitere Öffentlichkeit von AmateurPianistinnen und -Pianisten bestimmten Werken zu notieren beginnt.

Dabei werden in dieser Betrachtung die Solokonzerte weitgehend ausgespart, die in erster Linie für den eigenen Gebrauch des Komponisten und zugleich ersten Solisten bestimmt sind und in der Regel erst veröffentlicht werden, wenn sie sich nicht mehr aktuell in dessen Konzertrepertoire befinden. In diesen Stücken wird - nicht zuletzt ihrem virtuosen Charakter entsprechend - oft schon früher von einem Instrument mit größerem Tonumfang Gebrauch gemacht, zum Beispiel von einem Walter-Flügel mit zwei zusätzlichen Tasten fis'" und g'", der im Folgenden kurz als Walter plus bezeichnet wird.7 Dies zeigen auch die konzertanten Werke von Beethovens Zeitgenossen Anton Eberl (I765-I807): Sein Konzert C-Dur op.32 (komponiert spätestens I803, gedruckt I805), jenes in Es-Dur op. 40 (wahrscheinlich ebenfalls I803 entstanden und posthum I808 gedruckt) und das Doppelkonzert B-Dur op. 45 (1803/1809) $)^{8}$ verwenden durchwegs selbstverständlich das hohe g'" - ebenso wie bereits die frühen Notationsstadien des Klavierparts von Beethovens op. 37 schon vor dessen definitiver Erweiterung auf das c'"' hin: Aufgrund der Tonart c-Moll ist ohne g'" eine Darstellung des thematischen Materials dieses Werks auf dem Klavier undenkbar - oder dieses Material hätte anders ausgesehen, wenn es für ein bloß fünfoktaviges Instrument entworfen worden wäre. ${ }^{9}$

Eine umfassende Aufarbeitung der Frage nach dem tonsetzerischen Umgang mit sich ändernden Grenzen des Tonumfangs ist im Rahmen dieses Beitrags nicht möglich. Er beschränkt sich deshalb auf die Betrachtung einiger struktureller Besonderheiten in

6 Skowroneck bespricht etwa detailliert Fragen der Repetitionsgeschwindigkeit oder des una-corda-Pedals, vgl. ebd., S. IIIf. und S. 87-89.

7 Abbildung eines solchen Instruments bei Hecher: Designentwicklung, S. I8० (Abb.3): „Hammerflügel Anton Walter, Wien ca. I790, Kunsthistorisches Museum Wien, Sammlung alter Musikinstrumente, SAM 454."

8 Bert Hagels: Vorwort, in: Anton Eberl. Konzert C-Dur für Klavier und Orchester op. 32, Berlin 2008, S. III-V; ders.: Vorwort, in: Anton Eberl. Konzert Es-Dur für Klavier und Orchester op. 40, Berlin 2008, S. III-VI; ders.: Vorwort, in: Anton Eberl. Konzert B-Dur für zwei Klaviere und Orchester op. 45, Berlin 2008, S. III-V, hier S. IV (Anm. I5) auch der Hinweis auf die öffentliche Aufforderung an Eberl in der Allgemeinen musikalischen Zeitung, seine Konzerte drucken zu lassen: AmZ 7 (I804/05), Nr.33 (I5. Mai r805), Sp. 536.

9 Zu der bekannten Stelle in Beethovens (noch auf f'" beschränktem) Konzert C-Dur op. I5 (г. Satz, Takt I72), wo das im Vergleich mit Takt 387 erwartete fis"' eben noch nicht geschrieben wird, siehe Skowroneck: Beethoven the Pianist, S. 83 f. 
frühen Sonaten Beethovens aus der Zeit vor der Ankunft seines Érard-Flügels, die als kompositorische Auseinandersetzung mit dem dann zur Verfügung stehenden Tonumfang von lediglich fünf Oktaven interpretiert werden. Da es sich bei den meisten der zu besprechenden Stellen dieser Werke nicht nur um offensichtlich abgeschnittene einzelne hohe oder tiefe Töne handelt, sind sie auch großteils nicht dieselben wie die schon von John Henry van der Meer aufgelisteten. ${ }^{\text {IO }}$

Bevor aber dieser eigentliche Hauptteil der Betrachtung folgt und um Einblick in die mit dieser Fragestellung üblicherweise verbundenen kompositorischen Vorgangsweisen zu geben, wird anhand einer späten Klaviersonate von Wolfgang Amadeus Mozart der traditionelle Umgang mit dem Spitzenton f'" innerhalb des hergebrachten fünfoktavigen Tonumfangs im Sinne einer dadurch gestalteten formalen Dramaturgie vorgeführt. Ein Intermezzo bilden zwei Dokumente zu in diesem Zusammenhang signifikanten Übergangssituationen aus dem englisch-französischen Kontext: Johann Ladislaus Dussek lässt schon um 1795 in London in seinen Veröffentlichungen alternative kleine Noten »für Klaviere mit zusätzlichen Tasten« drucken, deren Bau er dabei selbst angeregt hat; in Paris um I8I5 dann sind es die zwar noch weiterhin in Gebrauch befindlichen, aber nicht mehr tonangebenden »kleinen« Klaviere alter Bauart, die mit Alternativstellen in Kleindruck bedacht werden.

\section{Beschränkung des Tonumfangs und Ökonomie im Gebrauch von Spitzentönen Mozarts}

Umgang mit den ihm im Laufe seines Lebens in erster Linie zur Verfügung stehenden Instrumenten Cembalo, Clavichord und Hammerklavier ist umfassend aufgearbeitet, ${ }^{\text {II }}$ und seine späten Werke für Tasteninstrument können gut als Musterbeispiele für den traditionellen kompositorischen Umgang mit dem fünfoktavigen Tonumfang $\mathrm{F}_{1}$ bis f'" auf höchstem Niveau dienen. Wofür Mozart in den späten r78oer-Jahren idealerweise komponiert hat, ist relativ klar: für ein Instrument in der Art seines eigenen Flügels von Anton Walter (I752-I826), den er I782 erwirbt und der sich heute im Mozart-Museum Salzburg befindet. Auch wenn es schon zu Mozarts Lebzeiten in seinem Umkreis vereinzelt Instrumente mit größerem Tonumfang gegeben haben mag, etwa den erwähnten Walter plus mit zwei zusätzlichen Tasten fis'" und g'", der bei einzelnen der Adressatinnen und Adressaten seiner Werke verfügbar gewesen sein wird, so halten sich seine Kompositionen (wie die quasi aller Wiener Komponistinnen und Komponisten noch die ganzen I79oer-Jahre hindurch) strikt an den traditionellen fünfoktavigen Umfang - umso mehr, wenn sie für eine Druckveröffentlichung bestimmt sind. 
Die einzige Ausnahme in Mozarts gesamtem Klavierwerk bildet die Sonate für zwei Klaviere D-Dur KV 448 (375a), die im 3. Satz ein einzelnes fis"' im ersten Klavier verlangt, was durch den privaten Charakter der Komposition erklärbar ist (sie wird erst posthum 1795 gedruckt)::22 Mozarts Schülerinnen Josepha Auernhammer und Barbara Ployer, mit denen er die Sonate bei verschiedenen Gelegenheiten im Rahmen privater >Akademien spielt, ${ }^{\mathrm{I} 3}$ verfügen wohl über ein Instrument mit zusätzlichen hohen Tönen - ein solches ist aber zu dieser Zeit nicht allgemein vorauszusetzen.

Die kompositorischen Strategien, die dazu dienen, die unverrückbare Beschränkung des offiziell bespielbaren Tonumfangs bis zum f'" in optimaler Weise produktiv zu gestalten, sollen hier anhand des ersten Satzes von Mozarts Klaviersonate KV 570 dargestellt werden, der

- in B-Dur und damit in einer für die vielfältige und problemlose Behandlung des Spitzentones f'" geeigneten und auch sehr verbreiteten Tonart steht,

- als konziser Repräsentant der `Sonatenhauptsatzform respektive >Form des ersten Allegros mit getreu eingerichteter Rekapitulation besonders aussagekräftig für die damit verbundenen handwerklichen Anforderungen zu sein scheint,

- zudem - da nicht von Mozart selbst für die Veröffentlichung vorbereitet - in allen wiederholenden Teilen unverziert und damit sozusagen nur gerüstartig überliefert ist, ${ }^{\mathrm{I} 4}$

- eine späte (im Februar I789 ins eigene Werkverzeichnis eingetragene) und damit gleichsam schon in den Zeitraum dieser Untersuchung hineinweisende Ausprägung darstellt, jedoch im Unterschied zu den anderen späten Sonaten Mozarts nicht erst I805, sondern

12 Sonate pour deux clavecins ou piano-forte [...] op.34, Wien: Artaria \& Comp. [I795] (PN 550); vgl. Alexander Weinmann: Vollständiges Verlagsverzeichnis Artaria \& Comp., Wien ${ }^{2}$ I978 (Beiträge zur Geschichte des Alt-Wiener Musikverlages, Reihe 2, Folge 2), S.39; Neue Mozart-Ausgabe [in Folge NMA] Ix, 24, Abteilung r: Werke für zwei Klaviere, Kritische Berichte, hg. von Ernst Fritz Schmid, Kassel u. a. I957, S. Io.

13 »Gestern war ich eben in der accademie beym Auernhammer [...] - wir haben das Concert à Due gespiellt, und eine Sonate in zweyen die ich expreß dazu Componirt habe, und die allen succès gehabt hat«. Mozart an seinen Vater, Salzburg [24. November 178I], in: Mozart. Briefe und Aufzeichnungen, hg. von der Internationalen Stiftung Mozarteum, Kassel u. a. I962, Bd.3: I780-I786, S. I76 f., hier S. I76; »Morgen wird bei H: Agenten Ployer zu döbling auf dem Lande Academie seyn, wo die frl: Babette ihr Neues Concert ex g - ich das Quintett - und wir beyde dann die grosse Sonate auf a Clavier spiellen werden.« Mozart an seinen Vater, Salzburg [9. Juni I784], in: ebd., S.317-319, hier S.318.

14 Siehe dazu Rampe: Mozarts Claviermusik, S. 285 f. (zum 2. Satz von Kv 570) und die ebd. angegebenen weiteren Beispiele; zur erwarteten Auszierung von Mozarts skizzenhafter Notation in den Klavierkonzerten siehe Leonardo Miucci: Mozart after Mozart. Editorial lessons in the process of publishing J. N. Hummel's arrangements of Mozart's piano concertos, in: Music \& Practice 2 (2015), www.music andpractice.org/volume-2/arrangements-of-mozarts-piano-concertos. 
- bereits 1796 in Wien gedruckt wird (als Teil einer dreiteiligen Werkgruppe zusammen mit den Violinsonaten $\mathrm{KV} 526$ und 48I, samt einer wohl deswegen durch den Verleger hinzugefügten Violinstimme $)^{15}$

- und von dem zumindest ein Teilautograph erhalten ist, dessen leicht zugängliches Faksimile einen direkten Einblick in Mozarts Niederschrift zulässt. ${ }^{\mathrm{I} 6}$

Die folgenden Überlegungen können anhand des in Notenbeispiel I synoptisch wiedergegebenen Verlaufs von Exposition und Rekapitulation direkt überprüft werden, wobei nur der in der Dominanttonart stehende Schlussteil der Exposition wiedergegeben wird, der in der Rekapitulation in die Grundtonart transponiert ein zweites Mal auftritt. Diese in jedem Sonatenhauptsatz vorgenommene Transposition wird im Folgenden nach Erwin Ratz »Einrichtung « genannt. ${ }^{\mathrm{I7}}$ Der hier nicht wiedergegebene Beginn des Satzes ist in Exposition und Rekapitulation gleichlautend (in Mozarts Autograph-Fragment bei der Wiederkehr deswegen nicht einmal ausgeschrieben) und enthält den Spitzenton f"' ebenso wenig wie die ganze Durchführung. ${ }^{\text {I8 }}$

Der in Notenbeispiel I wiedergegebene Teil besteht - jeweils in einer Tonart verbleibend - aus einem mit einem Quintabsatz (Halbschluss) endenden vorbereitenden Abschnitt, einem kontrapunktisch beginnenden Satz, der mit einem ersten Grundabsatz (Ganzschluss) schließt, sowie einem konzertant-passagenhaften Teil mit einer zweiten derartigen Kadenz, worauf noch eine wiederholt kadenzierende Schlussformulierung folgt. Diese Abschnitte werden im Folgenden schlagwortartig verkürzt bezeichnet als

Sonata per il Clavicembalo o Piano-Forte con l'accompagnamento d'un violino, Wien: Artaria \& Comp. [I796] (Plattennummer 663); Weinmann: Vollständiges Verlagsverzeichnis Artaria Q Comp., S. 42 (PN 628: KV 526, PN 629: KV 48I), S. 44 (KV 570); Vorwort, in: NMA IX, 25: Klaviersonaten, Bd. 2, hg. von Wolfgang Plath und Wolfgang Rehm, Kassel u.a. I986, S. viII-Xxiı, hier S. xviIIf.; NMA IX, 25: Klaviersonaten, Bd. I und 2, Kritische Berichte, hg. von Wolfgang Rehm, Kassel u. a. I998, S. I7I-I76.

16 Autographes Fragment mit dem Schluß des ersten Satzes (Faksimile), in: NMA IX, 25: Klaviersonaten, Bd. 2, S. $x x x f$.

17 Vgl. Erwin Ratz: Einführung in die musikalische Formenlehre. Über Formprinzipien in den Inventionen und Fugen J. S. Bachs und ihre Bedeutung für die Kompositionstechnik Beethovens, Wien 31973, S. 34.

18 Es geht hier nicht um eine >Formanalyse`, und so wird der wiedergegebene Abschnitt auch nicht mit den hergebrachten funktionalen Bezeichnungen >Überleitung «, SSeitengedanke $<$ und >Schlussgruppe versehen, die im I8. Jahrhundert kaum verwendet worden wären und die für diese Untersuchung keine Rolle spielen. Zur historischen Terminologie liegt eine Vielzahl an neuerer Literatur vor; hier sei nur auf die kurze Problemdarstellung bei Rampe: Mozarts Claviermusik, S. II5-I27 (Die Gattung Claviersonate), speziell S. I2If. (Die Form eines »ersten Allegros«) verwiesen. Um die beiden in Notenbeispiel I abgedruckten Teile pragmatisch miteinander vergleichen zu können, werden im Weiteren nur die beiden pauschalen Überbegriffe »Exposition« und »Rekapitulation« verwendet. 
- Vorbereitung (Takt 35-40/I65-I70),

- Satz (Takt 4I-57/I7I-I87) und (mit diesem verschränkt)

- Passagenteil (Takt 57-69/187-I99) sowie

- Schluss (Takt 70-79/200-209).

Betrachtet man zunächst den melodischen Verlauf der Exposition alleine (also das obere System von Notenbeispiel I), so fällt auf, dass der auf dem Instrument vorhandene Spitzenton f'" nur zweimal verwendet wird, nämlich einmal kurz vor der Kadenz am Ende des Passagenteils, die dadurch als Höhepunkt erscheint (Takt 67-69), das zweite Mal weniger im Fokus der Aufmerksamkeit - als variierte Wiederholung der ersten Teilphrase des Schluss-Abschnittes (Takt 75). Die Vorbereitung hält sich in einem mittleren Register, der ihr folgende Satz in einem hohen, mit einem Höhepunkt ebenfalls vor seiner Kadenz, dabei jedoch nicht den Spitzenton f'" berührend (Takt 54). Der Passagenteil beginnt tief, lässt einen möglichen Höhepunkt aus (Takt 62-65) und scheint schon in allertiefster Lage kadenzieren zu wollen, führt aber kurz vor der schon angesprochenen Kadenz in Takt ${ }_{7} \mathrm{f}$. die Melodie doch noch zum Spitzenton f'" hoch, um danach in mittelhoher Lage abzuschließen. Der Schluss hat einen von der hohen in die mittlere Lage absinkenden Gestus, und der höchste Ton f'" erklingt in ihm nochmals eher beiläufig.

Insgesamt kann von einem sehr bewusst geplanten und mit dem Spitzenton ökonomisch umgehenden dramaturgischen Verlauf des Endes der Exposition gesprochen werden, der auch der Interpretin oder dem Interpreten eine bestimmte pianistische Gestaltung des Spannungsbogens nahelegt, die in der Regel wohl intuitiv so ausgeführt wird, wie es der Weg durch die Register suggeriert. Ähnliche Vorgehensweisen lassen sich quasi in allen Klaviersonaten vergleichbarer kompositorischer Qualität der in Frage stehenden Zeit finden - KV 570 stellt in dieser Hinsicht also überhaupt keinen Sonderfall dar. Dass sich gerade die Grundtonart B-Dur (mit ihrer Dominanttonart F-Dur) sehr gut dafür eignet, in Exposition wie Rekapitulation das f'" als Höhepunkt zu präsentieren, liegt auf der Hand; in F-Dur/C-Dur funktioniert das ebenfalls problemlos, in Tonarten wie D-Dur/A-Dur hingegen müssen schon harmonische Kunstgriffe wie sekundärdominantische verminderte Septakkorde oder die Vermollung von Abschnitten in Betracht gezogen werden, um einen ähnlichen Umgang mit dem f'" praktizieren zu können, was den Tonsatz in solchen Fällen fast gezwungenermaßen harmonisch und tonal komplexer und vielschichtiger werden lässt. In dieser Weise kann die Aufmerksamkeit auf den Spitzenton nicht zuletzt einen Zugang für das Verständnis der bevorzugten Tonartwahl für Klaviermusik bieten.

Die melodische Linie der Rekapitulation des ersten Satzes von KV 570 (das untere System von Notenbeispiel I) weist einen von der Exposition abweichenden Spannungsverlauf auf: Ein erstes Mal wird das hohe f"' schon an das Ende der Vorbereitung gesetzt 


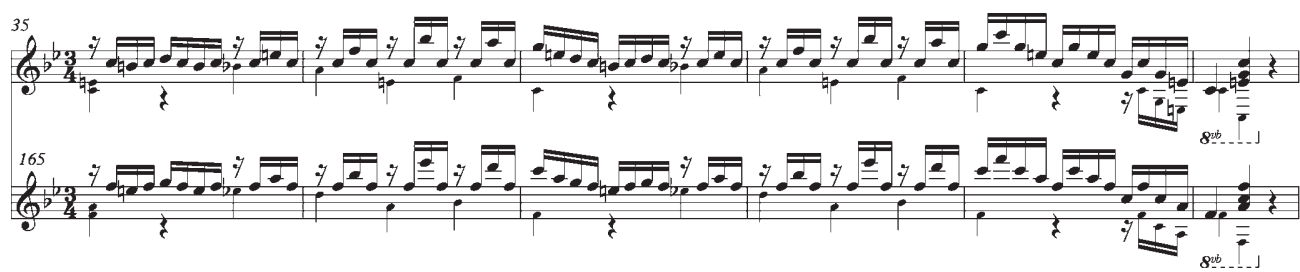

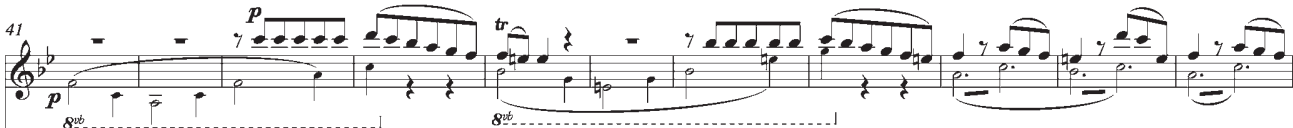

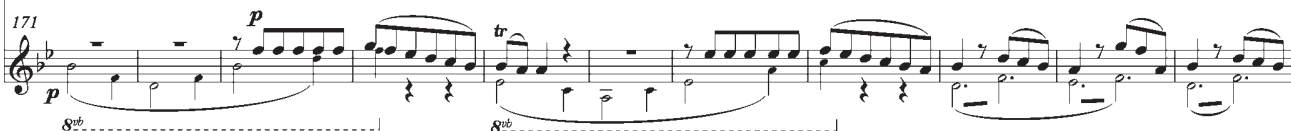

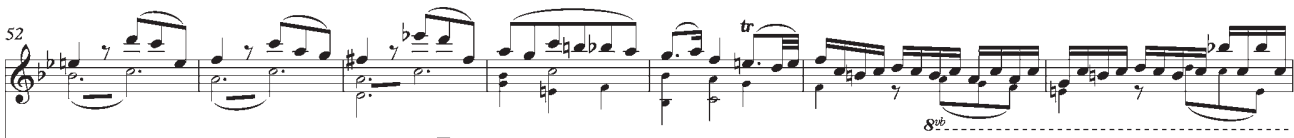

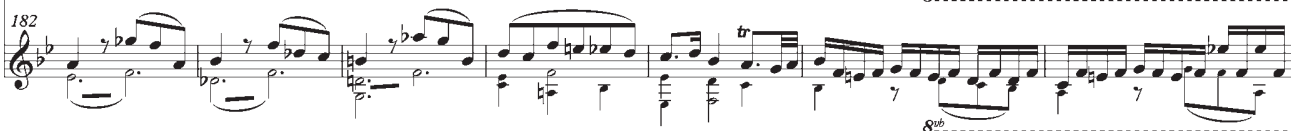

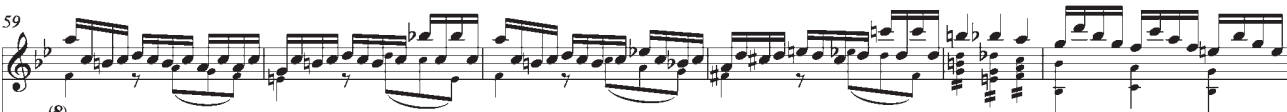

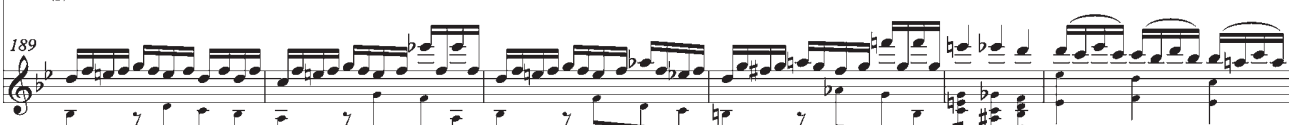
(8).
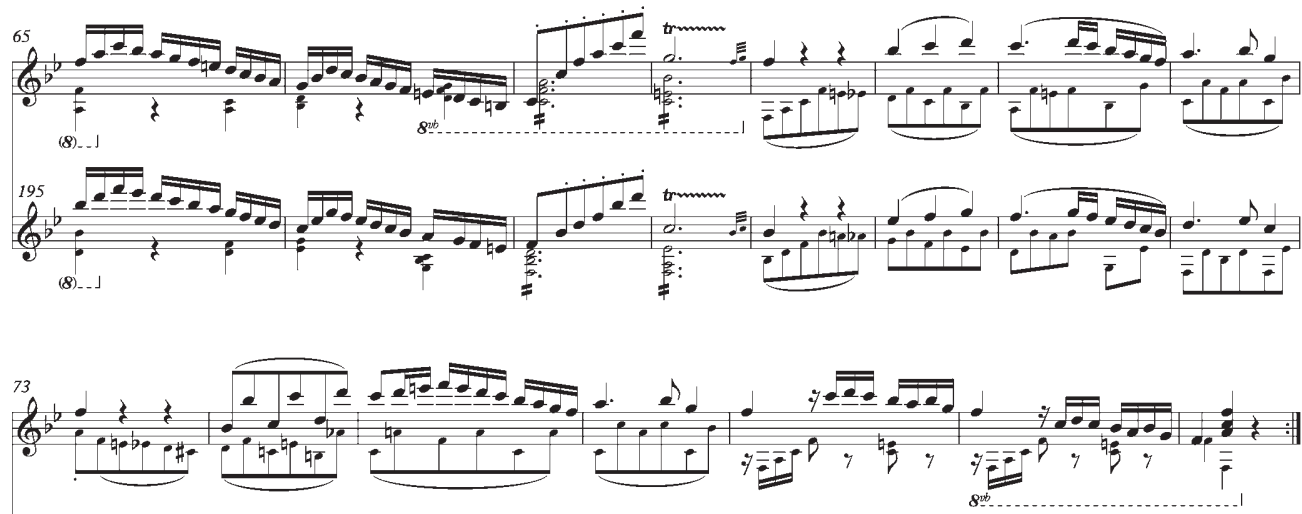

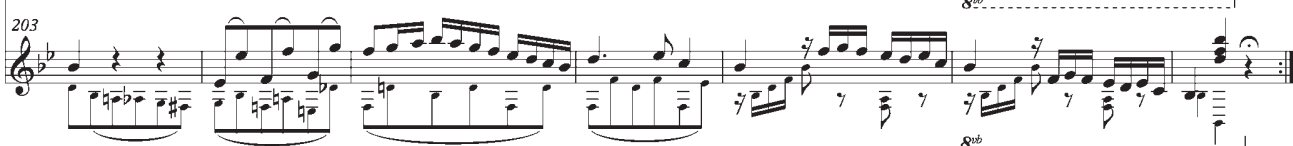

NOtE N B IS PIEL I Mozart: Klaviersonate B-Dur KV 570, 1. Satz: auf jeweils ein System komprimierte synoptische Darstellung des Schlussteils der Exposition (oberes System) und seiner Rekapitulation (unteres System). Die Oberstimme beider Teile ist in originaler Lage wiedergegeben; Oktavierungszeichen betreffen nur die klein gedruckten Unterstimmen. 
(Takt I69). Satz und Passagenteil liegen anfangs noch tiefer als in der Exposition, da sie bei Hochtransposition das f'" überschreiten würden; der Passagenteil wird allerdings mitten in seinem Verlauf hochoktaviert und kann in der Folge an genau der Stelle seinen Höhepunkt realisieren, wo ihn die Exposition zuvor ausgelassen hatte (in Takt I92-I95): Das f"' kommt einmal als oberer Eckpunkt einer steigenden harmonischen Sequenz und dann noch im solistischen Aufbäumen der melodischen Linie vor ihrem Niedersinken in die Kadenz vor. Der Schluss klingt in der Mittellage aus - schließlich soll das Stück damit unaufgeregt zu Ende gehen können.

Auch diese im Rahmen der Möglichkeiten (es handelt sich ja trotz allem immer noch um >dieselbe Musikı) auffallend unterschiedliche musikalische Dramaturgie der Rekapitulation in Kv 570 bildet keine Ausnahme, sondern ergibt sich aus der Sache selbst: Da die Rekapitulation gegenüber der Exposition um eine Quinte nach unten oder Quarte nach oben transponiert werden muss, kann das, was in der Exposition Höhepunkt war, nun keine vergleichbare Rolle mehr spielen. Die Kunst der Gestaltung einer Rekapitulation besteht darin, schon in der Exposition zu planen, welche ihrer Abschnitte nach oben und welche nach unten transponiert werden sollen: Dadurch entstehen neue Höhepunkte, während diejenigen der Exposition in weniger hohe Register zurückgestuft werden. ${ }^{\text {I9 }}$

In der Tabelle auf der nächsten Seite folgt ein Versuch, den Verlauf der beiden Formteile nochmals schematisch und auf die in diesem Zusammenhang wesentlichen Punkte reduziert zu visualisieren.

Im Zusammenhang der abschnittweisen Änderung der Transpositionsrichtung bleiben noch einige Stellen zu besprechen, deren meiste in der Tabelle mit einem Stern * bezeichnet sind:

- Es werden nicht immer beide Hände in dieselbe Richtung transponiert. Zu Beginn des Satzes ist die Hochtransposition der linken Hand wohl einfach klanglich begründet und erfordert aufgrund der Trennung ihrer Phrasen durch Pausen keine vermittelnden Änderungen (Takt I7I-I74).

- Zu Beginn des Schlusses wird der Abstand zwischen Ober- und Unterstimme um eine Oktave verringert (ab Takt I99); wenn dann in Takt 202 doch wieder der alte Abstand

19 Bei dem in Sonaten in Durtonarten üblichen Quint- (oder bei Hochtransposition Quart-)verhältnis von Exposition und Rekapitulation handelt es sich um das größtmögliche Intervallpaar, das dementsprechend den auffälligsten Einfluss auf den klanglichen Charakter des eingerichteten Abschnitts hat. Bei (Ende des I8. Jahrhunderts eher seltenen, aber im I9. Jahrhundert umso häufigeren) Sonaten in Moll-Tonarten bringt die Rücktransposition von der Paralleltonart zurück in die (dann oft verdurte) Grundtonart lediglich ein Sext- oder eben Terzintervall und damit die Möglichkeit größerer klanglicher Ausgeglichenheit mit sich. 


\begin{tabular}{|c|c|c|c|c|c|c|c|c|c|}
\hline \multirow{3}{*}{\multicolumn{2}{|c|}{$\begin{array}{c}\text { hoch } \\
\text { mittel } \\
\text { tief }\end{array}$}} & \multirow{2}{*}{\multicolumn{3}{|c|}{ Exposition }} & \multicolumn{2}{|l|}{ Vorbereitung } & \multicolumn{3}{|c|}{ Satz (bis $57 / 187 \rightarrow$ ) } \\
\hline & & & & & \multirow{3}{*}{\multicolumn{2}{|c|}{$\begin{array}{l}35-40 \\
\uparrow 165-170 \text { (169) }\end{array}$}} & \multicolumn{3}{|c|}{$41-48$} \\
\hline & & \multirow{2}{*}{\multicolumn{2}{|c|}{ Rekapitulation }} & \multirow{2}{*}{$\begin{array}{l}\text { r.H. } \\
1 . \mathrm{H} . \\
\end{array}$} & & & \multirow{2}{*}{\multicolumn{2}{|c|}{$\frac{\downarrow 171-174}{\uparrow 171-174}$}} & \multirow[t]{2}{*}{$\downarrow 175-178$} \\
\hline & & & & & & & & \\
\hline \multicolumn{3}{|c|}{$(\rightarrow)$ Satz } & \multicolumn{7}{|c|}{ Passagenteil (bis $69 / 199 \rightarrow$ ) } \\
\hline \multicolumn{3}{|c|}{$49-56$} & \multicolumn{4}{|l|}{$57-66$} & & & $67-68(67)$ \\
\hline \multicolumn{3}{|c|}{$\downarrow 179-186$} & $\downarrow 187-188$ & \multicolumn{4}{|c|}{$\uparrow 189-198(192) 194^{\star}(195)$} & \multicolumn{2}{|c|}{$197^{\star} \downarrow 198$} \\
\hline$(\rightarrow)$ & \multicolumn{5}{|c|}{ Schluss (ab 70/200) } & & & & \\
\hline \multicolumn{6}{|c|}{$69-76(75)$} & \multicolumn{2}{|l|}{77} & \multicolumn{2}{|l|}{$78-79$} \\
\hline \multicolumn{6}{|c|}{$\downarrow 199-206$} & \multicolumn{2}{|c|}{$\downarrow 207$} & $\downarrow 208$ & $\uparrow 209^{\star}$ \\
\hline \multicolumn{3}{|c|}{$\uparrow 199-201^{\star}$} & \multicolumn{3}{|c|}{$\downarrow 202-206$} & $\uparrow 2$ & & \multicolumn{2}{|c|}{$\downarrow 208^{\star}-209^{\star}$} \\
\hline
\end{tabular}

TA B E L L S Schematische Darstellung der Transpositionsprozesse aus Notenbeispiel 1 (Mozart: Sonate KV 570, 1. Satz, Ende von Exposition/Rekapitulation). Grauschattierung (Legende links oben): Einteilung der Oberstimmenkontur in relativ >hohe/mittlere/tiefe< Bereiche, $\uparrow \downarrow$ : Richtung der Transposition bei der Einrichtung, (Fettdruck: Takt mit Spitzenton f '"), *: Veränderung des Tonhöhenverlaufs gegenüber der Exposition. Die Taktzählung respektive Abschnittsgliederung entspricht hier nicht immer musikalischen Sinneinheiten (bei Verschränkung von Formteilen ist der die Phrase abschließende Schlusstakt nicht mitgezählt, sondern geht als Anfangstakt an die neue Einheit). Die ungefähre Einteilung in >hoch/ mittel/tief< betrifft in erster Linie die Oberstimme und ist bei der Unterstimme nur eigens vermerkt, wenn sie in die andere Richtung transponiert wird als die Oberstimme.

hergestellt werden soll, ist ein behutsamer Eingriff in die Basslinie nötig, der zu einer anderen harmonischen Lösung führt: Statt wie in der Exposition ausgehend von $\mathrm{I}^{6}$ stufenweise über $\mathrm{II}^{6}$ wird der Bass des in Takt 72/202 folgenden kadenzierenden Quartsextakkordes in der Rekapitulation von oben über $\mathrm{IV}^{6}$ erreicht - zugegebenermaßen ein geringfügiger Unterschied, aber angesichts der ansonsten fast durchgehend wörtlich transponierten Rekapitulation durchaus eine strukturelle Bruchstelle, die dabei elegant kaschiert wird.

- In den vier Abschlusstakten findet sich eine Anzahl von unauffälligen, aber effizienten kleinen Modifikationen: Auch hier wird zunächst der Abstand zwischen den beiden Stimmen um eine Oktave verringert (Takt 207), um dann gleich wieder den vorletzten Basssprung I-V(-I) um eine zusätzliche Oktave auseinanderzuziehen und ihm dadurch mehr Gewicht zu verleihen (was im nächsten Takt 208 ähnlich wiederholt wird, wodurch der auch in der linken Hand vorwiegend hohe Klaviersatz zumindest ganz am Ende einmal in einem tieferen Bassregister ankommt und somit gewissermaßen eine >Erdungく erfährt). 
- Ebenfalls als Schlusssignal zu interpretieren ist das einfache Höhersetzen des allerletzten Schlussakkords der rechten Hand um eine Oktave (Takt 209).

- Der Passagenteil weist die interessantesten strukturellen Eingriffe auf(schließlich soll er ja auch die Höhepunkte beider Versionen aufnehmen können): Wenn eine in der Einrichtung scheinbar aussichtslos immer tiefer rutschende Passage ruckartig um eine Oktave nach oben verschoben werden soll, muss der geeignete Ort für solch einen Schnitt gefunden werden - und was bietet sich dafür besser an als die Wiederholung der ersten beiden Takte des Passagenteils, die bei ihrem insgesamt vierten Auftreten ohnehin Gefahr laufen, redundant zu wirken (Takt 59/I89)? Hier wird die Unterstimme synchron mit nach oben genommen, was in dieser quasi orchestralen Passage in reizvoller Weise den Wechsel zu einem anderen Holzblasinstrument suggerieren mag.

- Nachdem fast der ganze Passagenteil nach oben transponiert worden ist, kommt sein Schlusstriller doch noch tiefer zu liegen als dessen Pendant in der Exposition (Takt 68f./I98f.). Der diese Änderung verursachende Eingriff in die Lagenstruktur und Stimmführung geschieht im Takt davor (Takt I97) und damit just an der Stelle, die in der Exposition dem Spitzenton f'" gewidmet ist (der auch dort mit einem abrupten Springen in die andere Richtung erreicht wird). Damit verbunden ist in Takt I97 die Abänderung des höchsten Tones der Oberstimme (in der Exposition f'" in Takt 67) zu einem d"', obwohl die getreue Transposition zu einem b" geführt hätte und in dem dort stehenden B-Dur-Quartsextakkord sogar das f'" ebenso gut möglich gewesen wäre wie zuvor im F-Dur-Quartsextakkord. Zum einen hat die Rekapitulation ihre Höhepunkte schon davor platziert (deswegen folgt hier kein weiteres f'"), zum anderen kommt es dadurch zu einer Modifikation der Oberstimmenbewegung: Der Schlusstriller steht nach wie vor auf der dafür vorgesehenen Melodiestufe (2), wird aber - statt eröffnend von (1) aus erreicht und anschließend wieder dorthin zurückkehrend ( $\mathrm{f}$ - $\mathrm{g}$ - $\mathrm{f}$, Takt ${ }_{7}$ ff.) - in einen abschließenden >Terzzug< (3) - (2) - (1) eingebunden ( $d$ - c - b, Takt I 97 ff.). ${ }^{20}$

- Zwischen den beiden f"' in Takt I92 und I95 findet sich in Takt I94 die auffälligste Änderung des melodischen Materials: Die Dreiklangszerlegungen der Exposition sind nicht wörtlich transponierbar (sie würden ein g"' erfordern) und werden in der Rekapitulation auf eine Terzenkette gekürzt sowie durch Vorhalte bereichert. Es kann die Hypothese gewagt werden, dass ein allfälliger Besitzer eines Walter plus mit zugreifen, verdankt sich einem Gespräch mit Stefan Rohringer im Jahre 2007 - damals auf die Sonate KV 333 (3I5c) bezogen, in deren ebenfalls in B-Dur stehendem I. Satz sich ganz ähnliche Vorgangsweisen finden (ab Takt 57/I59). 
umfangreicherer Klaviatur hier doch die Dreiklänge c"'- g'"- es'"'- c"' / b"- f'"'- d"'- b" / $a$ "- es"'- $c$ '"- $a$ " spielen (oder dies zumindest ernsthaft in Betracht ziehen und ausprobieren) sollte. Mozart kann eine solche wörtliche Transposition aus Respekt vor der Konvention des fünfoktavigen Klavierumfangs nicht veröffentlichen lassen (ganz abgesehen davon, dass er in eine von ihm selbst vorbereitete Druckveröffentlichung noch ganz andere Varianten und Verzierungen aufgenommen hätte), wird aber ein derartiges selbständiges Mitdenken der mit einem besonders guten Klavier ausgestatteten Interpretin wohl erwarten - genau dafür gibt es schließlich die beiden zusätzlichen Tasten fis'" und g"'! Außerdem entstünde dadurch zwischen den beiden f'" ein eindeutiger Höhepunkt des ganzen Satzes an einer passenden Stelle kurz vor Schluss.

Ganz allgemein ist noch zu sagen, dass die abweichenden Höhepunkte der Rekapitulation natürlich immer schon beim Schreiben der Exposition mitgeplant werden. Dabei ist jeweils das c'" entscheidend, das in der Rekapitulation zum f'" werden kann - Musterbeispiele in KV 570 finden sich in den Takten 39, 62 und 65. Takt 43 hingegen eignet sich nicht dafür, weil danach noch ein $d^{\prime \prime}$ folgt, das als transponiertes g'" nur auf einem Walter plus zur Verfügung stünde und vielleicht von einem gut gelaunten Mozart auf solch einem Instrument improvisatorisch ausprobiert worden sein wird (eventuell möchte sogar die besprochene Hochtransposition der linken Hand in Takt I7I-I74 genau dazu anregen?).

Zu welchen Eingriffen in Mozarts Text man sich als Interpret/in letztlich entscheidet (oder sie dann nach ihrem Ausprobieren doch wieder verwirft): Die am wenigsten sinnvolle Art, mit seiner Musik (und auch anderer) umzugehen, dürfte wohl darin bestehen, sie auf einem modernen Instrument mit viel größerem Tonumfang genau so zu spielen, wie sie in der Urtextausgabe festgehalten ist. >Interpretation « kann sich nicht nur auf die Frage beschränken, wie die immer gleichen Töne zu spielen sind - vielmehr umfasst ein wirkliches Verständnis ihres Ausgangspunktes Notation einen Einblick in die zugrunde liegenden kompositorischen Prozesse und sollte auch zum Überdenken dessen führen, was gespielt wird.

So viel zu elementaren kompositorischen Vorgangsweisen im Zusammenhang des Setzens von Höhepunkten jeweils gegen Ende von Formteilen, die im Einrichtungsverhältnis zueinander stehen und deren so unscheinbare wie kunstvolle Gestaltung die primäre Herausforderung für den Komponisten oder die Komponistin einer klassischen Sonate darstellt. 
Von »The Lines with the smaller Notes are for the Piano Forte with Additional Keys« (1795) zu »Les petites notes sont pour les petits pianos« (1814) Eine zentrale Rolle bei der Erweiterung des Tonumfangs des Klaviers wird Johann Ladislaus Dussek (I760-I8I2) zugeschrieben, der in der Zeit der Französischen Revolution seinen Lebensmittelpunkt für einige Jahre von Paris nach London verlegt. Er regt dort über den Druck eigener Werke den Bedarf nach größeren Klavieren an und beeinflusst gleichzeitig auch Klavierbauer:
"On 3I August I792 in St Anne's Church, Westminster, Dussek married Sophia Corri [...], who became famous as a singer, pianist, and harpist. During the remainder of his stay in London, he was associated with his father-in-law, Domenico Corri, in a music publishing business (Corri, Dussek \& Co.), which printed many of his works. While in London he also encouraged the firm of Broadwood to extend the range of the piano - in I79I from five to five and a half octaves, and in I794 to six octaves. Compositions written for the extended keyboard were said to be for ' piano with additional keys $;$ many compositions of this period were published with two versions for the right hand, so that they could be performed , with or without the additional keys $\star^{2 I}$

Für Dussek und seine Zielgruppe bildet der erweiterte Tonumfang neuer BroadwoodKlaviere noch die Ausnahme, und entsprechend werden die Passagen, die die alte Obergrenze f'" überschreiten, als Alternativen über das System der rechten Hand gedruckt so zum Beispiel in seinen Sonaten op. 25, die an den Stellen mit »additional keys« das erste Ausbaustadium der Klaviere von fünf auf fünfeinhalb Oktaven bis c'"' voraussetzen. ${ }^{22}$ Daneben gibt es auch Drucke, die direkt und ausschließlich »for the Grand \& Small Piano Forte with additional Keys« gedacht sind wie die Sonate B-Dur op. 24 (es werden nicht nur "große« Flügel, sondern auch »kleine« Tafelklaviere mit den zusätzlichen Tasten ausgestattet). ${ }^{23}$

An den Sonaten op. 25 bemerkenswert ist die editorische Sorglosigkeit der von Dusseks Schwiegervater Domenico Corri realisierten Londoner Originalausgabe (Abbildung I): Gleich der erste Ton der ersten Ossia-Stelle ist falsch als g"' statt f'" notiert und anschließend fehlt die all'ottava-Angabe.

21 Howard Allen Craw: Art. »Dussek family [Dusík, Dussik]«, (2) Jan Ladislav [Johann Ladislaus (Ludwig)] Dussek [Dusík], in: Grove Music Online, https://doi.org/Io.Io93/gmo/978I561592630.article.44229.

22 Three Sonatas for the Piano-Forte and also arranged For the Piano Forte with Additional Keps [...] Dedicated to the Right Honorable Lady Elizabeth Montagu, by J. L. Dussek, op. 25, London: D. Corri [1795] (Datierung nach Craw: Art. »Dussek family«). Exemplar aus Privatsammlung mit Vermerk »London, Printed by D. Corri, 28 Haymarket«; vgl. Exemplar GB-Lbl Music Collections g.452.e.: »Edinburgh, London: Printed for Messrs Corri, Dussek and Coy, [I794?]«.

23 A Sonata for the Grand \& Small Piano Forte With additional Keps, Composed § Dedicated to Mrs. Chinnerp By J. L. Dussek. Op. 24, London: Longman and Broderip [I793] (Datierung nach Craw: Art. »Dussek family«); Nachdruck in: Continental Composers in London I766-І8го, hg. von Nicholas Temperley, New York/London $\mathrm{I}_{985}$ (The London Pianoforte School I766-I860, Bd. 6), S. 75-84. 


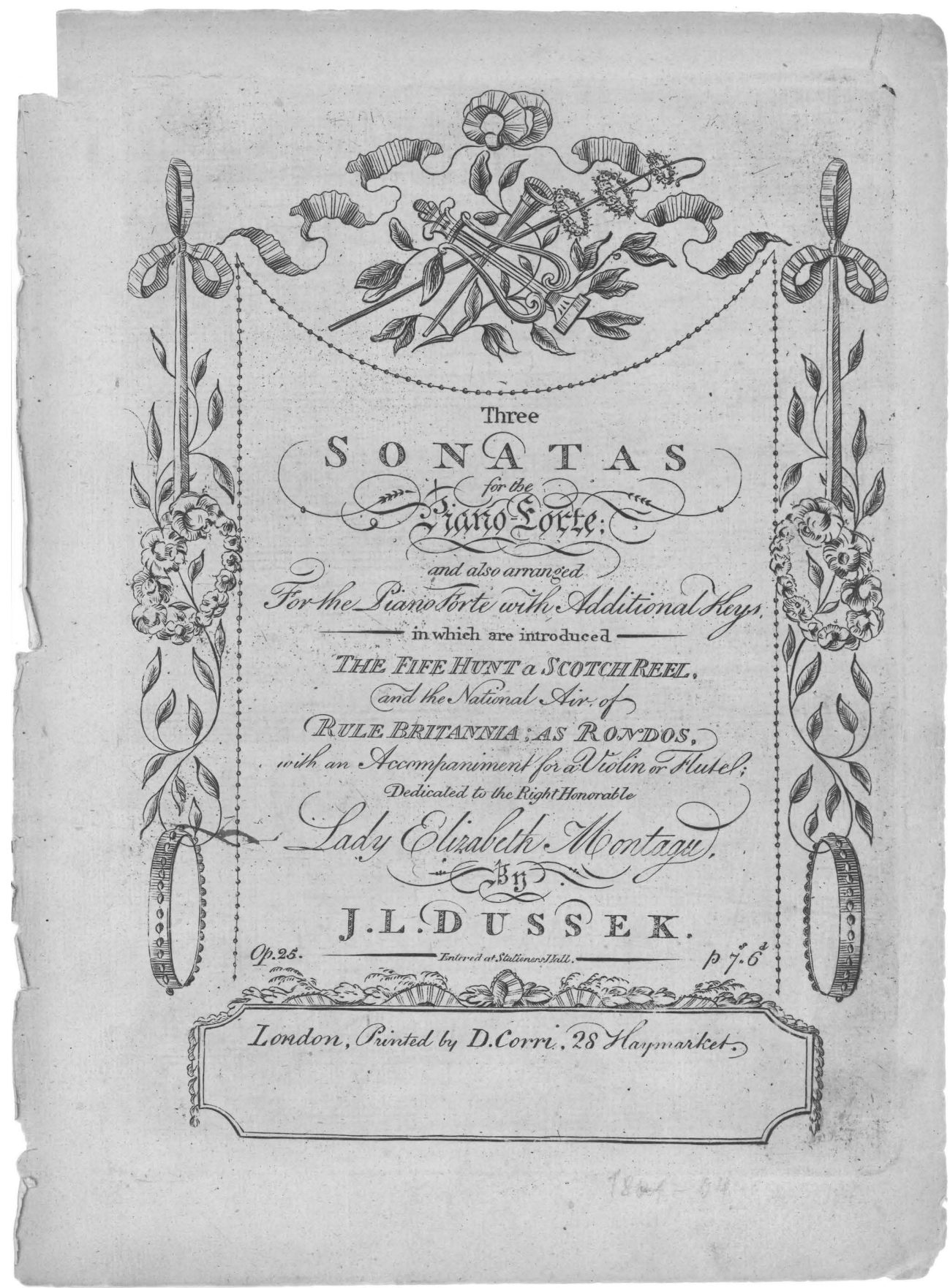

A B B ILDUNG I Titelseite von J. L. Dusseks Sonaten op. 25 in der Ausgabe Corri [1795] und Beginn des ersten Satzes von op. 25/1 mit Ossia-Noten »for the Piano Forte with Additional Keys«; Korrekturen und Fingersätze mit Bleistift von einer/-m frühere/n Besitzer/in (oder dessen/deren Lehrer/in), der oder die ein größeres Klavier zur Verfügung hatte. Bei den Fingersätzen bedeutet »+« den Daumen und »1-4« die weiteren Finger. 
19

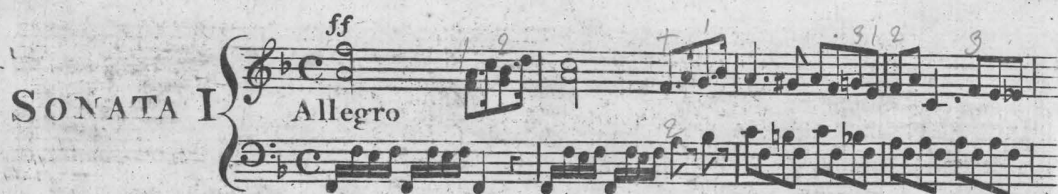

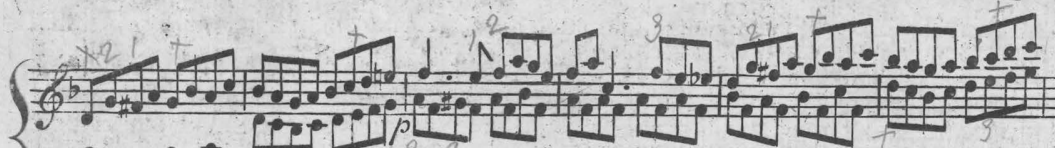

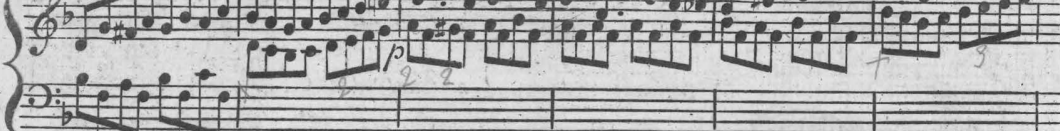
$\mathrm{d}^{\prime}$

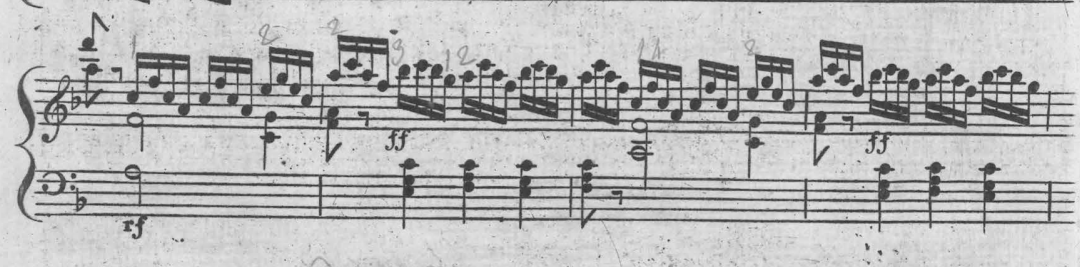

NB The Lines with the $f$ all

Notes are for the Piano Forte

With Additional Keys

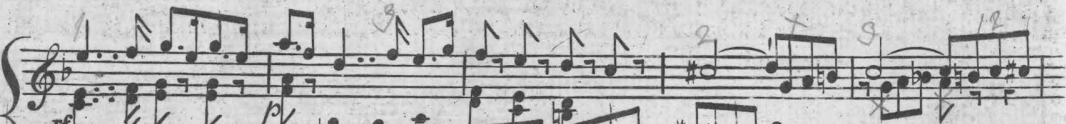

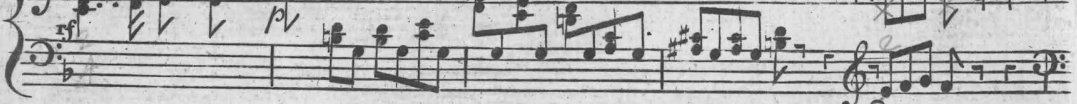

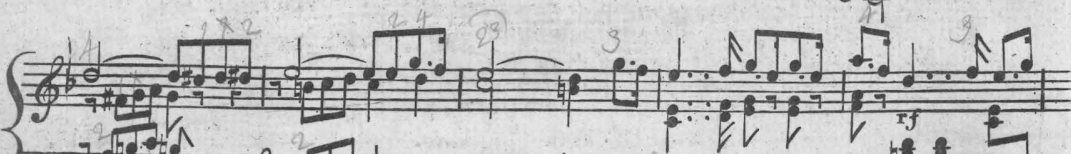

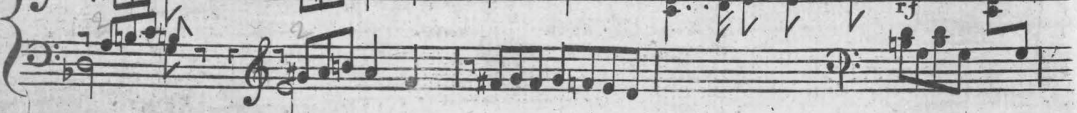


Eine wohl in zeitlicher Nähe zu Corris Druck veranstaltete und bis in dieZeilenumbrüche hinein mit ihm identische, aber neu gestochene Pariser Ausgabe von Sieber berichtigt diese Versehen, bringt den Hinweis auf die »additional keys « ebenfalls in englischer Sprache und fügt dem Notentext nur wenige Details hinzu (auf der in Abbildung I wiedergegebenen ersten Seite der Sonate op. 25/1 lediglich einen Doppelschlag im vorletzten Takt der letzten Zeile). ${ }^{24}$

Zwanzig Jahre später, kurz nach Dusseks Tod, als der Verlag Breitkopf\& Härtel in Leipzig zwölfbändige Oeuvres de J. L. Dussek herausbringt, ${ }^{25}$ ist die ehemalige Ossia-Version für größere Klaviere schon zur einzig wiedergegebenen normalen geworden, und keine der späteren Ausgaben dieser Werke hat den zwei Versionen bietenden Originaltext je wieder aufgenommen. ${ }^{26}$

Dass sich die Häufigkeit des Vorkommens der neuen Klaviere im Verhältnis zu den alten in der Zwischenzeit gewissermaßen umgekehrt hat, zeigt auch eine ungefähr zur selben Zeit wie Dusseks Leipziger Oeuvres in Paris erscheinende Sammlung von Klaviermusik verschiedener Autoren: Sie wird mit der Versicherung angekündigt, dass »[t]ous les morceaux composés pour les grands piano [sic] seront aussi arrangés pour ceux à cinq octaves «, ${ }^{27}$ und entsprechend ist dort der alternative Kleindruck für den geringeren Tonumfang reserviert (Abbildung 2): »Les Petites notes sont Pour les Petits Pianos. ${ }^{28}$

Auf S. I7 des Druckes (im I. Satz der Sonate op. 25/2) ist der Hinweis dann zusätzlich auf Französisch übersetzt: »Ces Lignes ajoutees en petites Notes ne se jouent que sur le Piano Forte a six octaves«. Trois Sonates Pour Clavecin Ou Forte Piano [...] Nota. elles peuvent être Exeéutés [sic] sur le Piano Forté a six octaves. Composeés et Dediés a Lady Elizabeth Montager [sic] par J. L. Dussek, CEuvre xxv, a Papis [sic] Chéz Sieber [... o. J.], Exemplar F-Pn VM7-8839, https://gallica.bnf.fr/ark:/12I48/btvib90674453. Der Katalogeintrag der Bibliothèque nationale de France datiert diesen Druck auf »1790«, obwohl auf seinem Titelblatt das »décret du I juillet I793 [relatif aux droits de propriété des auteurs d'écrits en tout genre, compositeurs de musique, peintres et dessinateurs]« erwähnt ist, vgl. http://catalogue.bnf.fr/ark:/ I2I $48 / \mathrm{cb}_{397} 840086$.

25 Oeuvres de J. L. Dussek, Cahier x: [...] Trois Sonates [...] op. 25, Leipzig: Breitkopf\& Härtel [1813-18I7], Datierung nach Nachdruck: Collected Works ofJan Ladislaus Dussek, New York I978, Bd. 5 .

26 Im International Music Score Library Project lassen sich aktuell neben der erwähnten vollständigen Ausgabe von Sieber noch verschiedene Drucke einzelner Sonaten oder Sätze von op. 25 aus der langen Geschichte des Pariser Verlags Lemoine und aus der Prager Serie Musica Antiqua Bohemica einsehen: https://imslp.org/wiki/3_Sonatas\%2C_Op.25_(Dussek\%2C_Jan_Ladislav).

27 Prospectus [= Subskriptionsanzeige], in: Mercure de France 55 (r. Mai I8I3), Nr. DCXv, S. 239 f., hier S. 240.

28 Walz par Mr. Paër, in: Recueil de walzes, allemandes et airs de différens caractères par les auteurs de la collection de pièces nouvelles pour le piano, Paris [ohne Verlagsangabe und Jahr], Suite de la Iz ème livraison, S. 6 f., hier S. 6. Exemplar aus Privatsammlung; vgl. dasjenige in I-PAc ML.32.7: http://id.sbn.it/bid/MUS oI25496: Erscheinen der Sammlung allerdings nicht wie dort angegeben »in der 2. Hälfte des I9. Jahrhunderts«, sondern wohl Ende I8I4, siehe die Anzeige im Mercure de France, S. 240: »La première livraison paraîtra dans le courant de janvier prochain, et les autres de mois en mois«, somit die vorliegende zwölfte im Dezember I8I4, falls dieser Editionsplan tatsächlich eingehalten worden ist. 


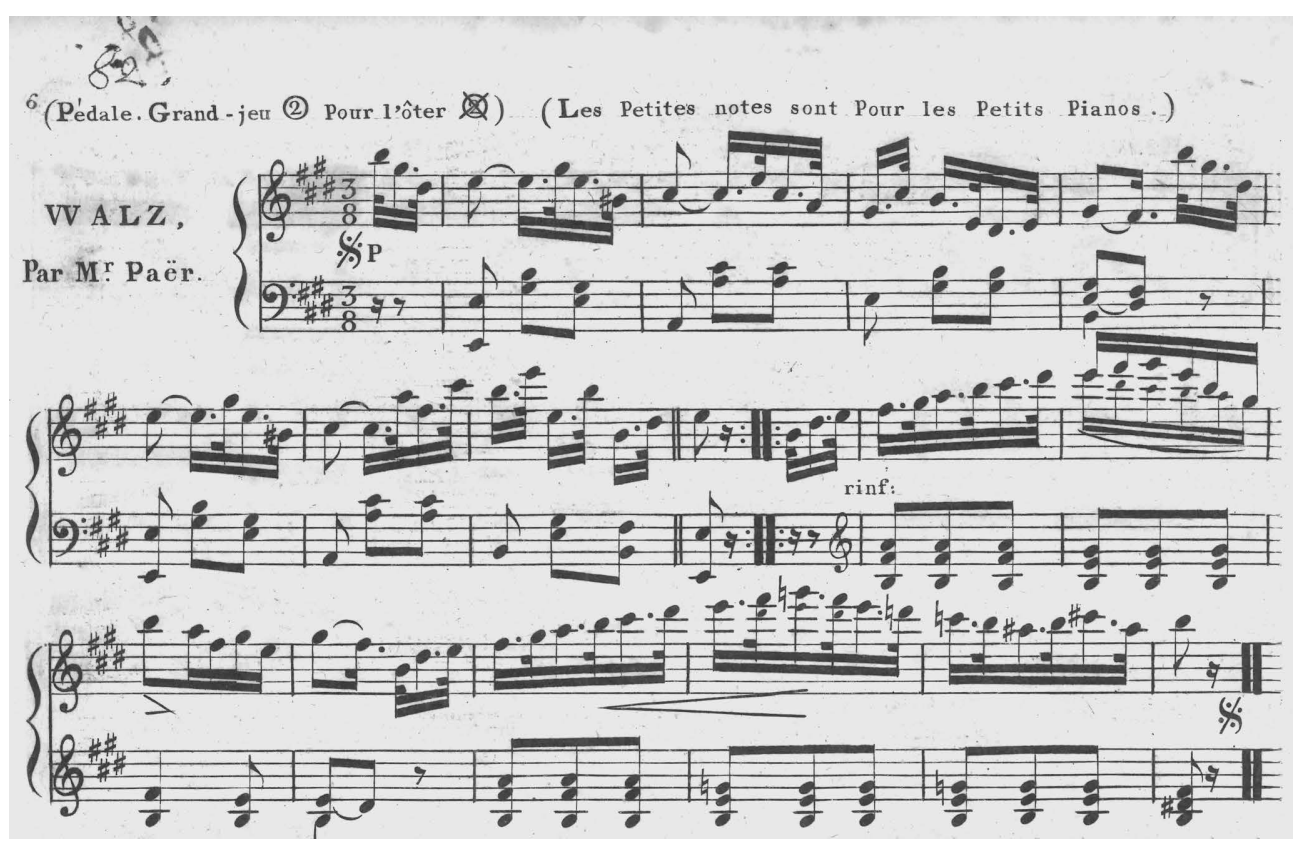

A B B ILDUNg 2 Beginn des Walzers von Ferdinando Paër aus Recueil de walzes ... [1814] mit kleinen Ossia-Noten für ältere Klaviere mit nur fünfoktavigem Tonumfang

Damit wird der Kreis der potentiellen Subskribentinnen und Subskribenten auch auf diejenigen ausgedehnt, die noch kein modernes größeres Klavier besitzen.

\section{Beethoven in der Zeit vor seinem Érard-Flügel: Deformation der kompositorischen Struktur} an den Grenzen des Klavierumfangs Anhand der besprochenen Sonate von Mozart haben wir aussagekräftige Beispiele gesehen für »subjects or motifs in the recapitulation of a sonata movement that were changed or compromised in comparison with the exposition in order to stay within a defined upper range «, ${ }^{29}$ um die Formulierung von Tilman Skowroneck aufzunehmen. Er meint die bereits erwähnte Beispielsammlung von van der Meer, ${ }^{3 \circ}$ wenn er weiter feststellt: »It is well established that Beethoven's earlier adherence to the five-octave compass resulted in many compromised textures and omitted notes in the treble and in the bass. We can, therefore, interpret the five-octave compass in Beethoven's oeuvre as a restriction. $\aleph^{\mathrm{I}}$ Allerdings haben wir es ja bei allen hier besprochenen Werken nicht mit fortschrittlich-genialisch-freien Kompositionen im Sinne einer späteren Zeit zu tun, die lediglich rigiden instrumentalen Grenzen unterworfen wären; vielmehr gehören sie immer noch einer Tradition an, die sich in erster Linie mit der

30 Van der Meer: Beethoven und das Fortepiano, insb. S. 59-64 (Beethovens Fortepianos in der Zeit bis I803).

31 Skowroneck: Beethoven the Pianist, S. I05 (Hervorhebung original). 
mehrfachen Verwendung von musikalischem Material in verschiedenen formalen Kontexten unter klar definierten tonartlichen Bedingungen beschäftigt. Schon angesichts von Mozarts souveräner Dramaturgie des Umgangs mit der höchsten Note f'" ist die Frage nicht von der Hand zu weisen, ob es wirklich angemessen ist, das kontrollierte und fantasievolle kompositorische Abschreiten des zur Verfügung stehenden Tonraums nur negativ als einer beeinträchtigenden Beschränkung unterworfen zu verstehen.

Wenn hier abschließend die Auseinandersetzung des jungen Beethoven mit den Grenzen des fünfoktavigen Klavierumfangs betrachtet wird, soll damit gezeigt werden, dass er dabei nicht nur >Kompromisse « macht, was die vielen abgeschnittenen tiefen und hohen Noten in seiner Klaviermusik, die van der Meer aufgelistet hat, durchaus nahezulegen scheinen; ${ }^{32}$ Beethoven nimmt vielmehr - so die hier vertretene Auffassung - diese Begrenzungen mitunter zum Anlass, kompositorische Konventionen in Frage zu stellen und ungewöhnliche Lösungen für die althergebrachte Herausforderung der rekapitulierenden >Einrichtung unter sparsamem Einbezug des höchsten zur Verfügung stehenden Tones vorzuschlagen. Und genauso wie später bei der Entgrenzung des Tonumfangs des Klaviers auch andere Parameter wie Tonartenplan, harmonischer Komplexitätsgrad und formale Ausdehnung in die Veränderung einbezogen werden, schlägt sich vor diesem großen Wandel die Reibung an der Beschränkung des Tonraums in einer umfassenden internen Umgestaltung der musikalischen Struktur nieder.

Wir kehren also zurück in die Zeit vor I803, in der zwar schon das eine oder andere Instrument mit größerem Tonumfang in Beethovens Umfeld vorhanden sein mag, aber gerade für Druckveröffentlichungen noch die ungeschriebene Regel gilt, den traditionellen fünfoktavigen Tonumfang des Klaviers nicht zu überschreiten.

- Der erste Satz der Sonate f-Moll op.2/I zeigt auf den ersten Blick die gewohnten Merkmale des ökonomischen Umgangs mit dem f"' gegen Ende von Exposition wie Rekapitulation. Da dieser höchste Ton jedoch in beiden Formteilen genau an der gleichen Stelle vorkommt - wie bei Mozart gesehen, angesichts der beiden verschiedenen Tonarten eigentlich ein Unding -, liegt hier ein Eingriff in die kompositorische Struktur vor (Takt 33/132, Notenbeispiel 2): Die drei ersten Töne der Oberstimme, die beide Male betont das f'" bringen, bevor sie sich mit der gleichen

Es gibt vergleichbare rabgeschnittene Stellen auch in anderer Instrumentalmusik, zum Beispiel wenn Beethoven in Takt I7 des ersten Satzes der Eroica-Sinfonie op. 55 der Flöte das problemlose Hervorbringen des hohen $b$ "' nicht zuzutrauen scheint und ihre Phrase deshalb früher enden lässt als die von Klarinette und Horn. Auch am Beginn des Streichquartetts C-Dur op. 59/3 würde die Basslinie im Cello eigentlich bis ins $\mathrm{H}_{1}$ führen, muss aber nach Erreichen der tiefsten leeren Saite $\mathrm{C}$ um eine Septime nach oben springen (vielen Dank für den Hinweis auf diese letztgenannte Stelle und ihr »virtuelles Überschreiten und Thematisieren dieser Grenze des Tonraumes« an Michael Lehner). 


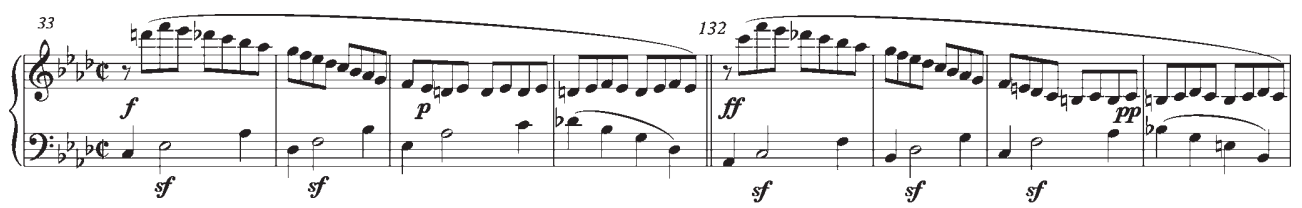

Noten be ISPIEL 2 Beethoven: Sonate f-Moll op.2/1, 1. Satz, Exposition/Rekapitulation

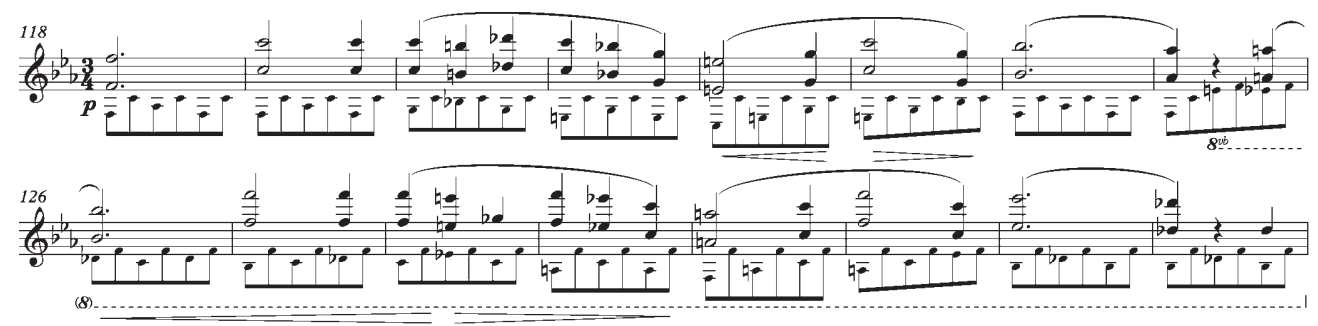

Noten beis PIEL 3 Beethoven: Sonate c-Moll op.10/1, 1. Satz, Durchführung. Oktavierungszeichen beziehen sich nur auf die klein gedruckte Bassstimme. Die folgenden Beispiele sind zum Teil auf Melodie und Bass reduziert.

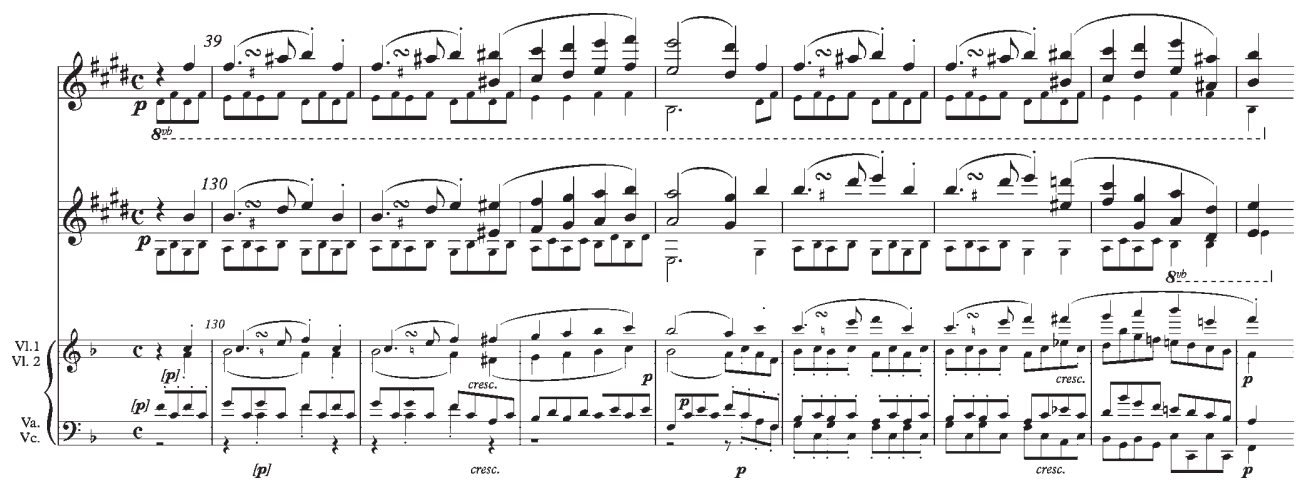

Noten B I I P I EL 4 Beethoven: Sonate E-Dur op.14/1, 1. Satz, Exposition (Klavierfassung oben) und Rekapitulation (Klavierfassung Mitte, Streichquartettfassung unten)

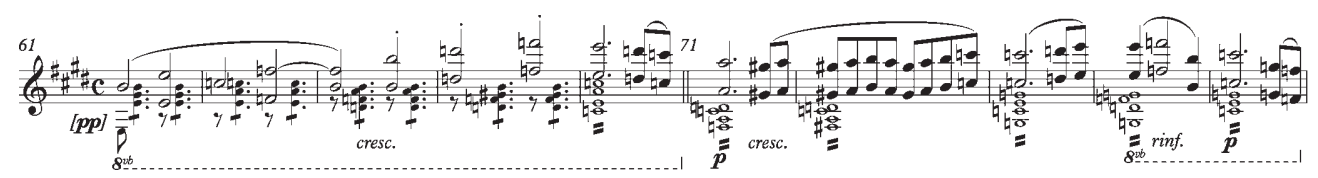

Noten beis PIEL 5 Beethoven: Sonate op.14/1, 1. Satz, Durchführung

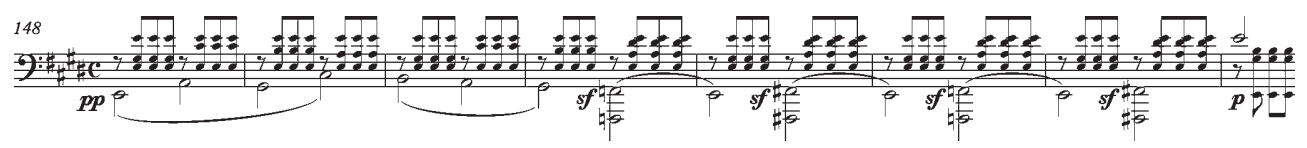

Noten BeISPIEL 6 Beethoven: Sonate op.14/1, 1. Satz, Coda 
Tonleiter nach unten bewegen, stellen in der Exposition in As-Dur die melodischen Stufen \#(4) - (6) - (5) dar (in Takt 37 (1) - (6) - (5)), in der Rekapitulation in f-Moll hingegen zweimal die Stufen (5) - (1) - (7). Beethoven schreibt also in den beiden Tonarten mit Ausnahme ihres ersten Tons gewissermaßen absolut die gleiche Melodie (was in einer Moll-Sonate mit ihrem Einrichtungsverhältnis Paralleltonart/Grundtonart weniger schwierig zu realisieren ist als in Dur mit der Dominanttonart) und deformiert dadurch die eigentlich zu transponierende melodische Struktur, was aber nicht sehr auffällt, weil diese genau dadurch bei ihrem zweiten Auftreten dem ersten noch ähnlicher ist als bei regulärer Transposition. All dies geschieht, um den traditionellen Höhepunkt wie gewohnt gegen Ende des Formteils bringen zu können.

- In der Durchführung des ersten Satzes der Sonate c-Moll op. Io/I geht Beethoven dazu über, knapp nicht verfügbare hohe Töne in seinen Tonsatz einzubeziehen, ohne sie dabei aber faktisch zu schreiben (Takt I28, Notenbeispiel 3): Dass in der Sequenzierung des in f-Moll stehenden Durchführungsmodells (ab Takt Ir8) in b-Moll ab Takt I26 ein ges'" gespielt werden müsste (und auf einem Walter plus ohne weiteres auch gespielt werden könnte), ist offensichtlich; allerdings steht es unbetont und piano mitten in einer insgesamt hohen und oktavierten Legato-Linie, dürfte also selbst im Falle seines tatsächlichen Erklingens nicht mit großem Nachdruck angeschlagen werden; umgekehrt fällt wohl auch sein Nicht-Erklingen gar nicht besonders ins Gewicht (oder es kann sogar als eine Art >Phantomton ₹ vermeintlich gehört werden), wobei das an dieser Stelle stark dissonante und obertonreiche harmonische Umfeld auch noch seinen Teil dazu beiträgt: Das im Dominantseptakkord mit einem es kombinierte $f$ wird durch seine Nebennoten $e$ und ges umspielt, man hört somit in b-Moll die jeweils einen Halbton auseinanderliegenden melodischen Stufen (4), \#(4), (5) und (6) quasi gleichzeitig.

- Im ersten Satz der Sonate D-Dur op. Io/3 wäre das nicht geschriebene fis'"' betont und fortissimo, kann hier aber als Oberton eines reinen Unisono-Satzes eventuell doch 'gehört` werden - und dass die höchsten Töne am schnellsten verklingen würden, versteht sich von selbst (Takt 22).33 Ganz ähnlich verhält es sich mit der Bewegung f"'- fis"'- g'"' in den jeweils ersten Sätzen der Violinsonate Es-Dur op. I2/3 (Takt 56 f.) und des »Gassenhauer «-Trios B-Dur op. II (Takt I57f.): Hier werden die ebenfalls fortissimo gespielten Unisono-Passagen des Klaviers durch die anderen beteiligten Instrumente Violine respektive Klarinette (oder Violine) und Violoncello in tieferen Oktavlagen mitgespielt und dadurch mit zusätzlichen Obertönen ausgestattet. In den Originalausgaben sind fis'" und g'" im Klavier schlicht nicht notiert; auf einem 
Walter plus hätte man sie sicher gespielt, auf einem fünfoktavigen Instrument sozusagen fast zu hören gemeint.

- Ein wie in op.ro/r unbetontes und durch Oktavierung gestütztes, also gewissermaßen en passant und piano zu spielendes fis"' wird im ersten Satz der Sonate E-Dur op. I4/I dann tatsächlich geschrieben und gedruckt, obwohl es von der Besitzerin oder dem Besitzer eines herkömmlichen Instrumentes höchstens suggestiv mitgedacht werden kann (Takt 4I, Notenbeispiel 4). An dieser Melodie des Seitensatzes und seiner Einrichtung lassen sich die durch Beethoven mitten in der Phrase angebrachten Wechsel der Transpositionsrichtung sowie weitere melodische Anpassungen studieren: Auf dem ersten Schlag von Takt I36 wäre in wörtlicher Einrichtung eigentlich ein fis"' am Platze gewesen, das aber - da betont - nicht gebracht wird. In der von Beethoven selbst arrangierten Version der Sonate op. I4/I für Streichquartett ${ }^{34}$ ist diese Stelle gegenüber der Klavierfassung verändert und regulär eingerichtet: Die I. Violine - gegenüber dem Klavier keiner unverrückbaren Obergrenze des Tonumfangs unterworfen - steigt dort bis ins b'" hinauf(Takt I36, Notenbeispiel 4, in F-Dur statt E-Dur).

- Im Bereich der Exposition/Rekapitulation, also in H-Dur/E-Dur, lässt sich ein f"' (oder auch ein eis"' ohne Weiterführung ins fis"') wirklich nur schwer anbringen; um also in einem Satz in E-Dur doch noch den traditionell höchsten Ton des Klaviers verwenden zu können, verfällt die Durchführung des ersten Satzes von op. I4/I darauf, sich in die etwas entfernteren Tonarten a-Moll und C-Dur zu begeben (Takt 64/ 74, Notenbeispiel 5). So lässt sich dieser >empfindsam ^oder gar >romantisch ‘ wirkende Moll-Einschlag des Satzes auch einfach als handwerklicher Kunstgriff der Lagendramaturgie interpretieren.

- In der Coda dieses Satzes findet sich noch ein schönes Beispiel dafür, dass das Weglassen des auf der Tastatur nicht vorhandenen tiefen $\mathrm{E}_{1}$ nicht immer nur Ergebnis eines widerwillig eingegangenen Kompromisses in der Hoffnung aufbaldige allgemeine Erweiterung des Tonumfanges sein muss, sondern durchaus einen musikalischen Sinn haben kann: Die sforzando gespielten und mit ihrer unteren Oktave versehenen abwechselnden tiefen Töne F und Fis können in ihrer jeweiligen Weiterführung ins dann wohl piano ausklingende E ohne großen Verlust oder sogar zu ihrem Vorteil dessen auf den Wiener Klavieren noch längere Zeit nicht vorhandene tiefe Oktave weglassen (ab Takt I5I, Notenbeispiel 6). Mit diesem Einsatz des tiefsten verfügbaren Tones $\mathrm{F}_{1}$ ganz am Ende des Stückes - in dessen GrundtonartE-Dur,in der er nicht leitereigen ist - kreiert Beethoven im Übrigen einen frühen Präzedenz-

34 Quatuor [...] d'après une sonate [...] par Louis van Beethoven arrangé par lui même, Wien: Bureau d'Arts et d'Industrie [1802] (PN I7); siehe dazu den Beitrag von Thomas Gartmann in diesem Band, S.379-398. 
fall einer übermäßigen Sexte auf der tiefalterierten Bassstufe (2), also zur Tonika hinführend (statt mit der tiefen (6) zur Dominante, wie bis dahin gleichsam ausschließlich üblich).

Dies sind einige Beispiele dafür, wie sich ein junger Komponist um I80o durch die Limitation des Tonumfangs zu ungewöhnlichen harmonischen Lösungen >zwingen lässt; abschließend sei noch ein Fall besprochen, in dem durch solche Unternehmungen die traditionelle Sonatenhauptsatzform einschneidend verändert wird: Der erste Satz der schon besprochenen c-Moll-Sonate op. Io/I bringt das hohe f'" ungewohnt früh (in Takt 6 f. und in der Schlusskadenz des Hauptsatzes Takt 28, Notenbeispiel 7) und gefährdet dadurch seine hergebrachte Funktion als allmählich vorbereiteter Höhepunkt gegen Ende eines Formteiles: Der mit diesem dramaturgischen Prinzip vertraute Hörer fragt sich somit schon ganz zu Beginn des Stückes, wie hier anschließend noch die gewohnte Steigerung erfolgen soll. Einerseits übertrifft Beethoven in der Durchführung den ehemaligen Hochton zwar wie besprochen mit einem Phantom-ges'", andererseits fällt aber der gewohnte Höhepunkt gegen Ende der Exposition schlicht aus: Hier wird lediglich mehrmals das es"' erreicht (das erste Mal mit einer langen Tonleiter in Takt 66, Notenbeispiel 8, oberes System), obwohl in der Paralleltonart Es-Dur, in der die Exposition schließt, durchaus ein f'" möglich gewesen wäre.

Die Rekapitulation des Satzes dann richtet diesen Abschnitt in völlig ungewöhnlicher und den vorgesehenen Tonartenplan in Frage stellender Weise ein: Der Seitensatz wird zuerst in F-Dur und somit einen Ton höher als in der Exposition gebracht (ab Takt 215, Notenbeispiel 8, mittleres System) und erst daran anschließend nochmals >korrekt $<$ in der Grundtonart c-Moll wiederholt (ab Takt 233, Notenbeispiel 8, unteres System). In der c-Moll-Version verschiebt sich der Höhepunkt auf den Quartvorhalt über der Tonika (Takt 239f.) - gegenüber dem entsprechenden Vorhalt über der Dominante in den beiden vorangegangenen Fassungen (Takt $66 \mathrm{f}$. beziehungsweise 225f.). Die Tonart F-Dur in der Rekapitulation ist (als in c-Moll nicht einmal leitereigen) schwer zu erklären - die hier vertretene Hypothese lautet, dass sie durch einfaches Höherrücken des Es-Dur der Exposition in der Rekapitulation 35 doch noch das Erreichen des f'" in diesem Formteil ermöglichen soll: Beethoven deformiert also an der oberen Tonraumgrenze nicht nur einzelne Motive (wie etwa in den von van der Meer zitierten Beispielen op.5/I und op.3r/3), ${ }^{6}$ sondern manipuliert grundlegende Parameter der Sonatenhauptsatzform, um

Es handelt sich um die gleiche Verrückung von Es-Dur nach F-Dur wie in der Rekapitulation des ersten Satzes der Eroica-Sinfonie Es-Dur op. 55 (ab Takt 408), für den sogar ein Wechsel der Stimmung des I. Horns von Es nach F mit Bogenwechsel für nur neun Takte verlangt wird. 


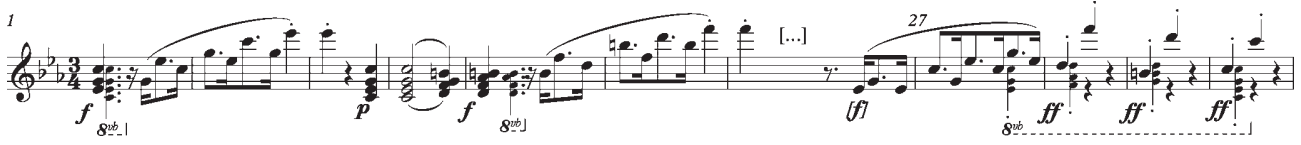

Noten B E IS PIEL 7 Beethoven: Sonate op.10/1, 1. Satz, Beginn

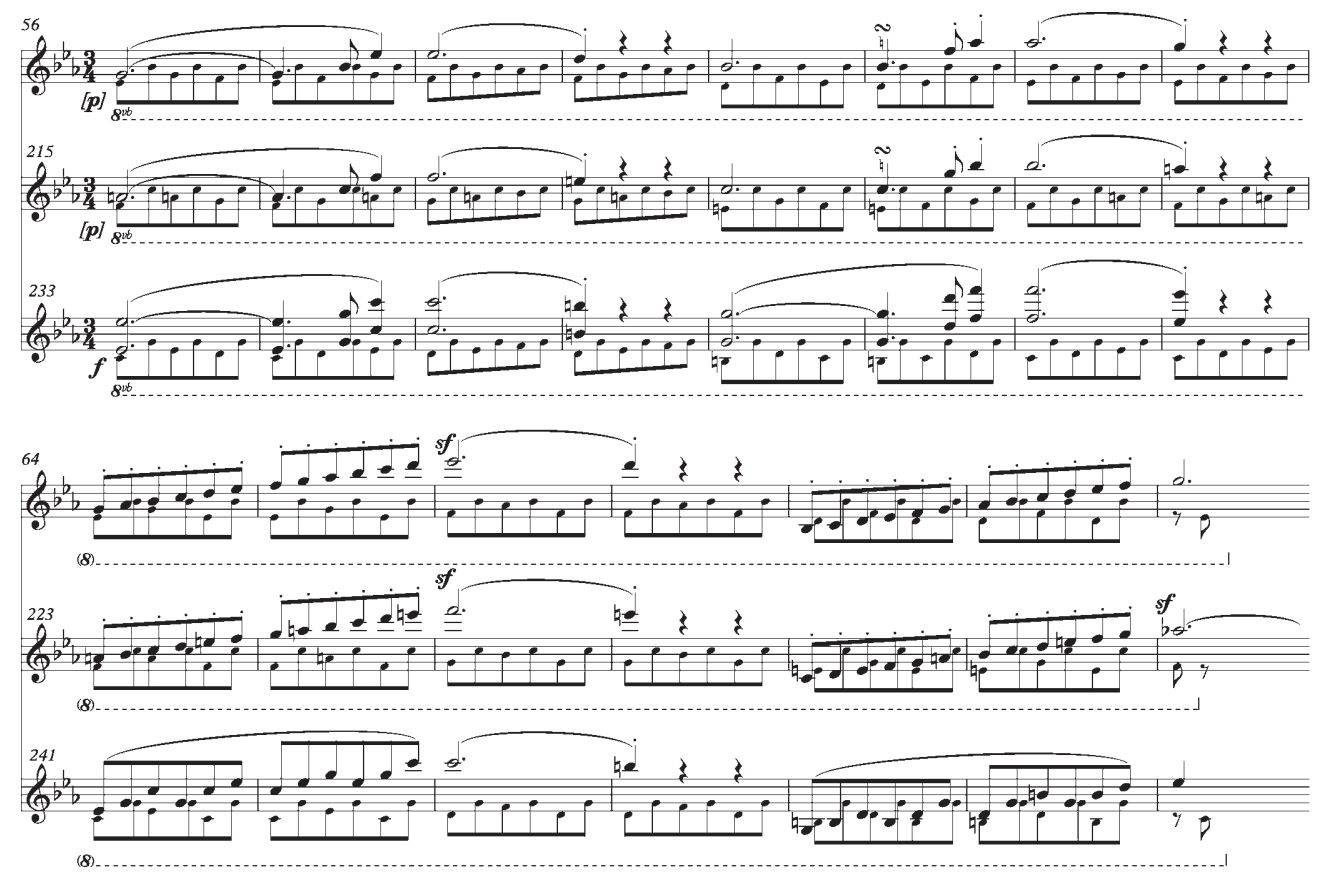

Noten beISPIEL 8 Beethoven: Sonate op. 10/1, 1. Satz, Exposition (oben)/

Rekapitulation (Mitte)/Weiterführung der Rekapitulation (unten)

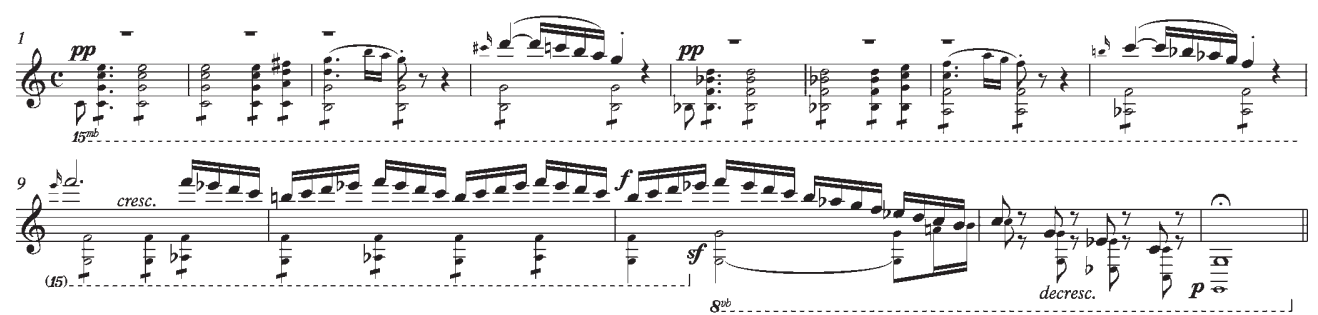

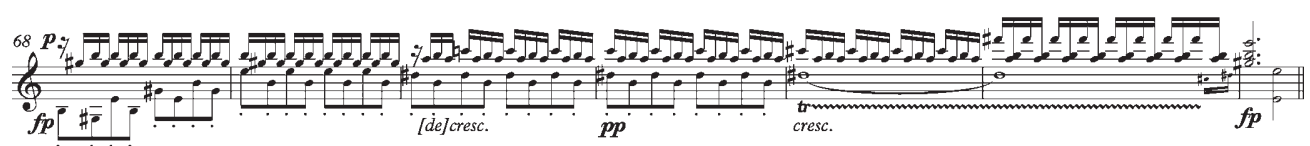

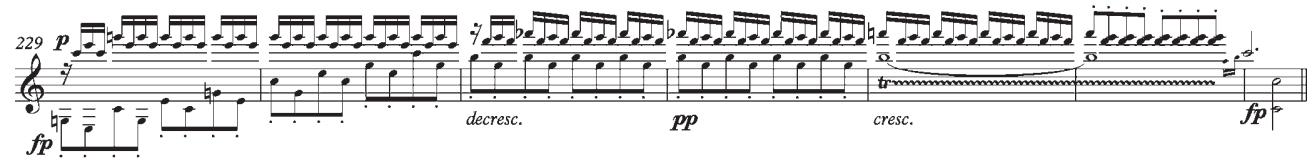

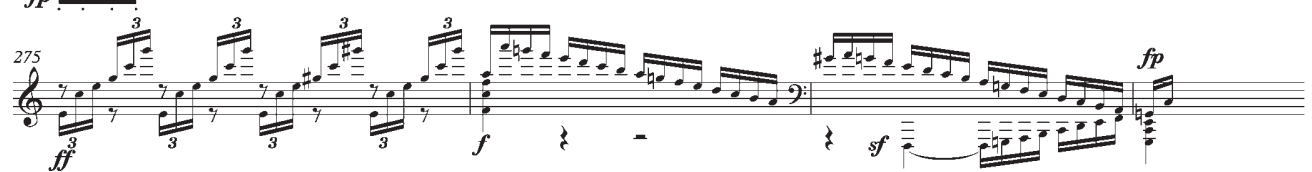

Noten beis PIEL 9 Beethoven: Sonate C-Dur op.53, 1. Satz: Beginn/Exposition/Rekapitulation/Coda 
sein Material optimal in den immer noch streng fünfoktavigen Tonumfang des Klaviers einpassen zu können.

Mit der wohl vorbildhaft wirkenden Präsenz des Érard-Flügels bei einem tonangebenden jungen Komponisten in Wien ab I803 und der damit verbundenen Aussicht auf eine weitere Verbreitung von Instrumenten mit größeren Tonumfängen gerät innerhalb weniger Jahre eine rasante Entwicklung in Gang, die an dieser Stelle nur noch angedeutet werden kann: Die Waldstein-Sonate C-Dur op. 53 kann das f'" schon ganz zu Beginn gewissermaßen >verschwenden (Takt 9 ff., Notenbeispiel 9), weil sie es danach mehrmals übersteigen wird - wenn auch nur bis zum a"', welchem Umstand Tilman Skowroneck eine ausführliche und differenzierte Betrachtung gewidmet hat.37

Wenn man seiner Analyse noch etwas hinzufügen will, so höchstens einige genuin tonsetzerische Überlegungen: Es ist richtig, dass in Takt 234 als Einrichtung von Takt 73 (Notenbeispiel 9) anstelle des a"' eigentlich ein d'"' und damit ein sogar den Umfang des Érard-Flügels übersteigender Ton stehen müsste; trotzdem greift es zu kurz, hier nur einen »compromise for reasons of keyboard compass« zu sehen, denn die ganze Stelle wird durch diesen Eingriff - wohl mit Absicht - harmonisch fragwürdig. Die gegenüber dem in allen Stimmen regulär weitergeführten Quintsextakkord der Exposition (Takt 73f.) hier zu einem Septnonenakkord in Umkehrung (beziehungsweise zu einem Quintsextakkord mit hinzugefügter Septe) angereicherte Dominante, die ohne jegliche Stimmführung in die leere Oktave der Tonika fällt (Takt 234f.), hinterlässt bewusst einen vorläufigen und unbefriedigenden Eindruck: Das kann es hinsichtlich der Behandlung des Spitzentons a'" noch nicht gewesen sein! Deswegen folgt in der Coda des Satzes (Takt 275 f., Notenbeispiel 9 - auch diese Stelle ist von Skowroneck besprochen und reproduziert) ein weiterer und sinnvollerer Anlauf, diesen Ton anzusteuern - diesmal über den Sextakkord $I^{6}$ mit hochalteriertem Quintton. Das a"' passt als Terz der dadurch erreichten IV. Stufe (Takt 276) besser als vorher über der $V_{5}^{6}$ (Takt 233f.), außerdem durchläuft diese Stelle innerhalb von zwei Takten sicher nicht zufällig nochmals den gesamten zur Verfügung stehenden Tonumfang des Klaviers bis hinunter zum $\mathrm{F}_{\mathrm{I}}$ (Takt 277).

Insofern ist Beethovens »Zögern, neue Klavierumfänge voll auszunützen«, $3^{8}$ in der Regel (und gerade in op. 53) durchaus rational erklärbar: Die hier vertretene These lautet, dass Beethoven jetzt zwar selbst einen Flügel zur Verfügung hat, der bis zum c'"' hoch funktionieren würde, ihn aber dennoch in dieser Sonate ganz bewusst zunächst nur bis zum a'" verwendet - vielleicht, um fürs erste einmal die Besitzerinnen und Besitzer der eingangs erwähnten Schantz-Flügel mit diesem Tonumfang zu überzeugen oder auch 
schlicht den Wiener Klavierbauern nicht allzu anspruchsvolle Vorgaben für die weitere Entwicklung ihrer Instrumente zu machen. Diese Erweiterung ins a"' komponiert Beethoven in geradezu programmatischer Weise chromatisch als g'"- as'" / gis"'- a"' aus (und baut damit auf dem virtuellen f'"- fis"'- g"' älterer Werke wie op. II und op. I2/3 auf). In der Rekapitulation fällt diese Bewegung in der dargestellten Weise zunächst absichtlich >verunglückt< oder zumindest sprovisorisch < aus (Takt 233 f.) und erscheint erst in der Coda sin korrigierter und definitiver Version< (Takt 275f.). Diese gewissermaßen rhetorische Vorgangsweise einer anfangs als Problem dargestellten und erst in der Coda gelösten >selbstgestellten Aufgabe ‘ findet sich bezüglich verschiedener Parameter in vielen von Beethovens frühen Werken.

Dieser Entwicklungsschritt der Erweiterung des Tonumfangs verläuft aufjeden Fall nicht geradlinig (die Sonate op. 54 geht wieder nur bis f'", während zum Beispiel op. 57 den ganzen neuen Umfang bis zum c'"' ausnutzt), und es muss nach wie vor zumindest teilweise Gegenstand von Vermutungen bleiben, für welche konkreten Instrumente Beethoven komponiert hat. Aufjeden Fall stellt Skowroneck mit guten Argumenten ein Fragezeichen hinter »a tradition in Beethoven studies that presupposes a direct and tight connection between Beethoven's instruments and the specific keyboard ranges that appear in his music «.39 Die in diesem Beitrag angestellten Betrachtungen sollten ansatzweise gezeigt haben, dass sogar das noch viel weiter verbreitete simple Fortschritts-Paradigma hinterfragt werden muss, durch dessen Brille der alte fünfoktavige Tonumfang nur negativ als »restriction « wahrgenommen wird - entwickeln doch einige von Beethovens frühen Klavierwerken idiosynkratische Strategien dafür, diese Beschränkung durchaus produktiv zu nutzen.

Besonders interessant sind diese Fragen im Zusammenhang von Beethovens verschiedenen Projekten von Neu- und Gesamtausgaben seiner eigenen Werke, die ihn in der Korrespondenz mit wechselnden Verlegern über fast zwei Jahrzehnte beschäftigen. ${ }^{40}$ Dabei ist nämlich immer wieder von einer geplanten Veränderung und Aktualisierung des Notentextes die Rede: So schreibt Beethoven I8ıo an Breitkopf\& Härtel: »Ich würde jeden Bogen [...] genau nachsehen, hie \& da Veränderungen anbringen, kurz meinerseits alles mögliche beitragen ein richtiges, correctes \& permanentes Werk zu liefern «. ${ }^{4 \mathrm{I}}$ Hätte Beethoven seine für den fünfoktavigen Tonumfang bestimmten frühen Klavierwerke

39 Ebd., S. ro5.

40 Siehe dazu Leonardo Miucci: Ignaz Moscheles' Ausgaben von Ludwig van Beethovens Klaviersonaten. Philologische und aufführungspraktische Perspektiven, Bonn (Schriften zur Beethoven-Forschung), Kapitel I.I: Die Editionsprojekte von Ludwig van Beethoven (I803-I826) und I.2: Die Gesamtausgabenprojekte nach dem Tod des Komponisten (in Vorb.).

41 Beethoven an Breitkopf \& Härtel in Leipzig [Anfang August I8ıo], in: Ludwig van Beethoven. Briefwechsel Gesamtausgabe, hg. von Sieghard Brandenburg, Bd. 2, München I996, S. I46-I48, hier S. I47. 
samt ihren auf diese Begrenzung zurückzuführenden strukturellen Eigenheiten somit später geändert? Generell wird in solchen brieflichen Verhandlungen die Herausgabe älterer Werke - wenn überhaupt - nur als rentabel betrachtet, wenn gleichzeitig eine Vereinbarung zur möglichst exklusiven Überlassung neuer Kompositionen zustande kommt. Aufjeden Fall werden Eingriffe in den Notentext stets als Anlass für ein höheres Honorar für den Komponisten thematisiert, und der Antwort von Breitkopf \& Härtel an Beethoven I8Io ist zu entnehmen, dass diese Veränderungen - wenn sie denn je vorgenommen worden wären - auch schlicht als Rechtfertigung dafür hätten dienen sollen, überhaupt eine Neuausgabe zu veranstalten: »Ihre Werke hingegen sind das Eigentum verschiedener Verleger geworden, welche eine neue Ausgabe als eine Beeinträchtigung ihres frühern Rechts ansehen würden, wenn nicht diese Werke merklich verändert erschienen wären. $\aleph^{42}$ Anton Diabelli stellt ı8ı6 Überlegungen zur Honorierung Beethovens »für die öffentliche Autorisation zur Herausgabe Ihrer ClavierSachen, und zugleich für die Redaction davon « an, ${ }^{43}$ und ein Schreiben Beethovens an Simrock I8I7 legt nahe, dass bei noch einem anderen Gesamtausgabenprojekt gegenüber den Originalausgaben und ihren Nachdrucken auch schlicht Fehler zu korrigieren gewesen wären: »Es wäre ein in mancher Hinsicht erklekliches Unternehmen, da so viele Fehlervolle Ausgaben meiner Werke in der welt herum spazieren «.44

Derartige Neuausgaben früher Klavierwerke in einer Revision durch Beethoven selbst sind nie zustande gekommen, und spätere Herausgeber scheinen den Notentext bereits avant la lettre als das unantastbare »neue [...] Testament des Klavierspielers « $45 \mathrm{zu}$ betrachten, verändern ihn also auf keinen Fall (zumindest nicht in Hinsicht auf die zu spielenden Töne, sondern lediglich etwa durch zusätzliche Artikulations-, Pedalisierungs- und Vortragsbezeichnungen). Ganz konkret zur nötigen Überarbeitung der ursprünglich für den fünfoktavigen Umfang komponierten Stücke äußert sich nur der mit Beethoven befreundete Klavierbauer Johann Andreas Streicher im Herbst I824 in einem Brief an den Komponisten:

»Wenn Sie in der Ankündigung bemerken daß Sie r) alle Clavier-Stüke, welche vor Einführung der Pianoforte von $5^{1 / 2}$ oder 6 octaven, geschrieben worden, hie und da umändern und nach den jetzigen Instrumenten einrichten wollen, wenn Sie 2) den ClavierSachen auch einige Neue, ungedrukte Stüke beyfügen, so ist diese Herausgabe wie ein ganz frisches, neu componirtes Werk zu betrachten, und muß auch von demjenigen gekauft werden, der Ihre früheren Werke schon besitzt. Die Sache selbst kann Ihnen unmöglich so viele Mühe machen, daß Sie solche nicht unternehmen könnten. Sie

Breitkopf\& Härtel an Beethoven (24. September 1810), ebd., Bd. 2, S. 155-I60, hier S. 157.

43 Anton Diabelli an Beethoven (22. August 1816), ebd., Bd.3, S. 284f., hier S. 284.

44 Beethoven an Peter Joseph Simrock [I5. Februar I8I7], ebd., Bd. 4, S. 27 f., hier S. 28.

45 Diese Äußerung von Hans von Bülow wird erst 1880 gedruckt: Auserlesene Klavier-Etüden von Fr. Chopin, mit Vorwort, Anmerkungen und Fingersatz von Hans von Bülow, München: Aibl [I88०], S. [I]. 
sind dieses sich selbst, Ihrem Neffen, für den Sie dann leichter etwas thun können, und der Nachwelt schuldig.« $4^{6}$

Auch dem Verleger Peters gegenüber, mit dem Beethoven zu dieser Zeit in Verhandlungen steht, wiederholt Streicher im selben Sinne: »Diese Werke würden aber nicht nur so, wie solche schon öffentlich erschienen, herausgegeben, sondern verändert, den jetzigen Pianoforte angepaßt, und fast zu jedem Hefte, ein neues Stück gegeben werden.«47

Offensichtlich einfach sabgeschnittene tiefe und hohe Töne zu ergänzen, die auf moderneren Klavieren verfügbar sind, hätte keine große Änderung bedeutet; wie jedoch die dargestellten Modifikationen der Oberstimme oder sogar des Tonartenverlaufs in den frühen Klaviersonaten in sinnvoller Weise für größere Tonumfänge hätten eingerichtet werden sollen, ist entgegen der Annahme Streichers doch eine schwierigere Frage.

Die schon angesprochene Bearbeitung der Sonate op. I4/I für Streichquartett(Notenbeispiel 4) kann Anhaltspunkte dafür geben, wie Beethoven möglicherweise dabei vorgegangen wäre: In der Coda ihres abschließenden Rondos findet sich ein Eingriff in die Satzstruktur, der dabei nicht einmal durch den Tonumfang bedingt ist (ab Takt III, Notenbeispiel ıo). Könnte man daraus schließen, dass Beethoven in seiner allfälligen Überarbeitung des Satzes für eine Neuausgabe der Klaviersonate analog zur Streichquartettfassung die Dominante in der Mitte des Taktes Iı2 nicht in Septlage arretiert, sondern auf die Oktave hin überstiegen, also die anschließend abwärts führende Tonleiter auf dem Ton h begonnen (und ab dem fis im nächsten Takt zusätzlich oktaviert) hätte?

Eine Reparatur der >falschen Einrichtung in der Rekapitulation der Sonate op. Io/I schließlich (ab Takt 2I5, Notenbeispiel 8), ohne durch sie den Ablauf des Stückes völlig zu verändern, scheint vollends unvorstellbar.

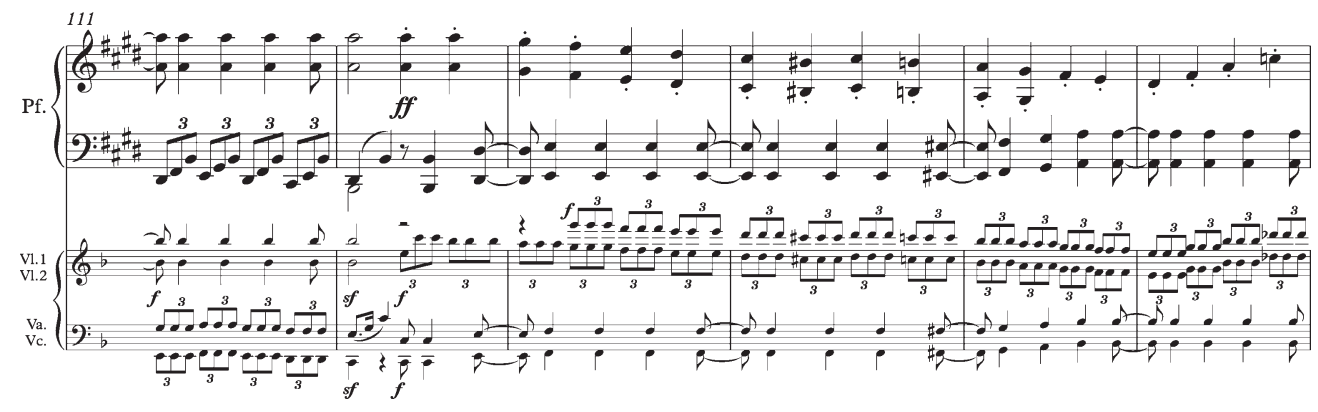

Noten B E I S P IEL 10 Beethoven: Sonate op. 14/1, 3. Satz, Takt 111-116:

Fassung für Klavier (oben), für Streichquartett (unten)

46 Johann Andreas Streicher an Beethoven (5. September 1824), in: Ludwig van Beethoven. Briefwechsel Gesamtausgabe, Bd. 5, S. 358-360, hier S. 359 f.

47 Johann Andreas Streicher an Carl Friedrich Peters (25. September 1824), ebd., Bd.5, S.37I-373, hier S. 372 . 
Auch wenn die genannten Projekte von Gesamtausgaben wohl in erster Linie an

- den Rechtsansprüchen der Originalverleger,

- Beethovens Honorarforderungen, damit

- der fragwürdigen Rentabilität für den neuen Verlag sowie nicht zuletzt

- am Anspruch, zu jeder Gattung noch ein neues Werk zu liefern,

gescheitert sein dürften: Mit dem Ziel einer modernisierten oder gar >definitiven $<$ Neufassung von älteren, in je eigener Weise zeitgebundenen und instrumentenbezogenen Werken sind nicht zu unterschätzende satztechnische Aspekte verbunden. Wie das fast beliebig gewählte Beispiel aus der Streichquartettfassung der Sonate op. I4/I zeigt, hätte sich hier sehr schnell ein möglicherweise allzu weites Feld von Änderungsmöglichkeiten aufgetan - wenn nicht der entscheidende, so doch mit ein Grund dafür, dass die Projekte von überarbeiteten Neuausgaben nie zustande gekommen sind und Beethoven stattdessen lieber neue Stücke komponiert hat. 


\section{Inhalt}

Vorwort 8

INTERPRETATION - BEGRIFF, METHODE, PRAXIS

Laure Spaltenstein Interpretation als treue Übersetzung. Zur Frühgeschichte eines vieldeutigen Begriffs I5

Kai Köpp Von der Quelle zur Methode. Zum Entwurf einer historischen Interpretationsforschung $\quad 28$

Manuel Bärtsch >Interpretation‘. Beethovens Sonate A-Dur op. IOI in der Sicht von Eugen d'Albert und Frederic Lamond

Sebastian Bausch Klavierrollen als Interpretationsdokumente. Ein Erfahrungsbericht als Leitfaden für Einsteiger $\quad 7 \mathrm{I}$

Camilla Köhnken Beethoven-Auslegung zwischen Liszts »Deklamationsstil« und Bülows »Vivisektionsversuchen«. Auf den Spuren Liszt'scher Interpretationsideale in Hans von Bülows instruktiver Edition der Klaviersonaten Beethovens $\quad 92$

Neal Peres Da Costa Carl Reinecke's Performance of his Arrangement of the Second Movement from Mozart's Piano Concerto K. 488. Some Thoughts on Style and the Hidden Messages in Musical Notation

II4

Carolina Estrada Bascuñana Enrique Granados's Performance Style.

Visualising the Audible Evidence I5O

Lukas Näf Tempogestaltung in Weberns Sinfonie op. 2I I80

INTERPRETATION - AUFFÜHRUNGSGESCHICHTE

Christoph Moor "Ein so erklärtes Lieblingsstück der hiesigen Kunstfreunde«. Die Rezeptionsgeschichte der Jupiter-Sinfonie in Beethovens Wien

Luisa Klaus Objektive Bruckner-Interpretation? Zur Aufführung der Trio-Entwürfe für die Neunte Sinfonie I940 205

Chris Walton Von innen und von außen. Beethovens Neunte Sinfonie und die $>$ Wagner'sche< Dirigiertradition $\quad 2 \mathrm{I} 8$

Lena-Lisa Wüstendörfer Streit um Fidelio. Gustav Mahler und Felix Weingartner im Disput um Werktreue $\quad 238$

INTERMEZZO

Robert Levin Turning Point to Musical Modernity. Beethoven as Executor of the Legacy of C. P. E. Bach. Concert Lecture 249

INTERPRETATION - INSTRUMENTE, ANALYSE, EDITION

Martin Skamletz «Man hat diese Erweiterung des Tonumfanges seit ein paar Jahren an den Tasteninstrumenten sehr weit getrieben.« Der Umgang mit Grenzen beim späten Mozart und beim frühen Beethoven $\quad 263$ 
Stephan Zirwes Analyse und Interpretation. Adolph Bernhard Marx' Beethoven-Analysen 29I

Michael Ladenburger Was können wir aus Originalhandschriften von Beethoven für eine angemessene Interpretation lernen? 30I

Federica Rovelli Die Skizzenbuch-Ausgaben und ihre mögliche digitale Zukunft 3 I7

Johannes Gebauer Interpretationspraktische Stemmatik. Philologische Methoden in der Interpretationsforschung am Beispiel annotierter Notenausgaben von Rodes 24 Capricen und Beethovens Violinkonzert

John Rink Chopin Copying Chopin 349

Tomasz Herbut Alexander Goldenweiser und Beethovens Sonate op. IIo- eine Spurensuche 366

INTERPRETATION - KREATIVE ANEIGNUNG

Thomas Gartmann Beethoven als sein eigener Interpret. Gedanken zur Bearbeitung der Klaviersonate op. I4/ז für Streichquartett

Ivo Haag Die Sinfonien von Johannes Brahms - (auch) Klaviermusik?

Michael Lehner Das Orchester auf dem Klavier. Welte-Klavierrollen von Gustav Mahler und Richard Strauss als interpretationsanalytische Quellen

Roger Allen “That Is What Music Really Is". Richard Wagner's Reception of Beethoven's Piano Sonata in A Major Op. IOI $43 \mathrm{I}$

Daniel Allenbach Eine heroische< Neunte? Dmitri Schostakowitschs Neunte Sinfonie im Vergleich mit Ludwig van Beethoenvs Sinfonien Nr.3 und 9 44I

Simeon Thompson Beethoven und der Zweite Weltkrieg in der künstlerischen Reflexion der Nachkriegszeit. Stanley Kubricks A Clockwork Orange und Rolf Liebermanns Leonore 40/45 456

Michelle Ziegler Rettungsversuch im Jubiläumsjahr. Mauricio Kagels Aufarbeitung der Beethoven-Rezeption in der Ludwig van-Werkgruppe (I970) 465

Leo Dick Über den späten Beethoven zur >Postidentität،. Die Suche nach liminalen Räumen im gegenwärtigen Musiktheater am Beispiel von Matthias Rebstocks Berliner Produktion Büro für postidentisches Leben $\quad 476$

Elizabeth Waterhouse Choreographic Re-mix. William Forsythe's Trio (I996) and Beethoven's String Quartet No. I5 in a Minor Op. I32 487

László Stachó "Gradus ad Parnassum".

The Purgatory of Instrumental Technique

Namen-, Werk- und Ortsregister 522

Die Autorinnen und Autoren der Beiträge 


\section{Rund um BeEthoven \\ Interpretationsforschung heute • \\ Herausgegeben von Thomas}

Gartmann und Daniel Allenbach 


\section{MUSIKFORSCHUNG DER Hochschule der KÜnste Bern Herausgegeben von Martin Skamletz und Thomas Gartmann Band 14}


0 Dieses Buch ist in gedruckter Form im Dezember 2019 in erster Auflage in der Edition Argus in Schliengen/Markgräflerland erschienen. Gestaltet und gesetzt wurde es im Verlag aus der Seria und der SeriaSans, die von Martin Majoor im Jahre 2000 gezeichnet wurden. Gedruckt wurde es auf Eos, einem holzfreien, säurefreien, chlorfreien und alterungsbeständigen Werkdruckpapier der Papierfabrik Salzer im niederösterreichischen Sankt Pölten. Das Vorsatzpapier Caribic cherry wurde von Igepa in Hamburg geliefert. Rives Tradition, ein Recyclingpapier mit leichter Filznarbung, das für den Bezug des Umschlags verwendet wurde, stellt die Papierfabrik Arjo Wiggins in Issy-les-Moulineaux bei Paris her. Das Kapitalband mit rot-schwarzer Raupe lieferte die Firma Dr. Günther Kast aus Sonthofen im Oberallgäu, die auf technische Gewebe und Spezialfasererzeugnisse spezialisiert ist. Gedruckt und gebunden wurde das Buch von der Firma Bookstation im bayerischen Anzing. Im Internet finden Sie Informationen über das gesamte Verlagsprogramm unter www.editionargus.de, zum Institut Interpretation der Hochschule der Künste Bern unter www.hkb.bfh.ch/interpretation und www.hkb-interpretation.ch. Die Deutsche Nationalbibliothek verzeichnet diese Publikation in der Deutschen Nationalbibliografie; detaillierte bibliografische Daten sind im Internet über www.dnb.de abrufbar. (c) der zeitgleich erschienenen digitalen Version: die Autorinnen und Autoren, 20I9. Dieses Werk ist lizenziert unter einer Creative Commons Namensnennung-Nicht kommerziell 4.0 International Lizenz (CC BY-NC 4.o). DoI: https://doi.org/I0.26045/kp64-6I78 ISBN 978-3-93I264-94-9 